\title{
Gluon transversity in polarized proton-deuteron Drell-Yan process
}

\author{
S. Kumano ${ }^{1,2,3, *}$ and Qin-Tao Song ${ }^{1,3,4, \uparrow}$ \\ ${ }^{1}$ KEK Theory Center, Institute of Particle and Nuclear Studies, High Energy Accelerator Research \\ Organization (KEK), Oho 1-1, Tsukuba, Ibaraki, 305-0801, Japan \\ ${ }^{2} J$-PARC Branch, KEK Theory Center, Institute of Particle and Nuclear Studies, KEK, and Theory Group, \\ Particle and Nuclear Physics Division, J-PARC Center, Shirakata 203-1, Tokai, Ibaraki, 319-1106, Japan \\ ${ }^{3}$ Department of Particle and Nuclear Physics, Graduate University for Advanced Studies (SOKENDAI), \\ Oho 1-1, Tsukuba, Ibaraki, 305-0801, Japan \\ ${ }^{4}$ School of Physics and Microelectronics, Zhengzhou University, Zhengzhou, Henan 450001, China
}

(Received 28 October 2019; accepted 14 February 2020; published 10 March 2020)

\begin{abstract}
Nucleon spin structure functions have been investigated mainly by longitudinally polarized ones for finding the origin of the nucleon spin. Other types of spin structure functions are transversely polarized ones. In particular, quark transversity distributions in the nucleons have very different properties from the longitudinally polarized quark distribution functions, especially in scaling violation, because they are decoupled from the gluon transversity, due to the fact that they are helicity-flip (chiral-odd) distributions. Such studies are valuable for finding not only the origin of the nucleon spin but also a signature on physics beyond the standard model, because the electric dipole moment of the neutron is proportional to the transversity distributions. Now, there is experimental progress on the quark transversity distributions; however, there is no experimental information on gluon transversity. In fact, the gluon transversity does not exist for the spin- $1 / 2$ nucleon due to the helicity-conservation constraint. One needs a hadron with spin more than or equal to one, so that the helicity flip of two units is allowed. A stable spin-1 target is, for example, the deuteron for studying the gluon transversity. In this work, we propose a possibility for finding the gluon transversity at hadron-accelerator facilities, especially in the proton-deuteron Drell-Yan process with the linearly polarized deuteron, by showing theoretical formalism and numerical results. In the experiment, the information on the angular distribution of the dimuon is necessary in the final state; however, the proton beam does not have to be polarized. We show the dependencies of the Drell-Yan cross section on the dimuon-mass squared $M_{\mu \mu}^{2}$, the dimuon transverse-momentum $q_{T}$, the dimuon rapidity $y$ in the center-of-momentum frame, and the magnitude of the gluon transversity $\Delta_{T} g$. We also show typical spin asymmetries in the Drell-Yan process. Since the internal spin- $1 / 2$ nucleons within the deuteron cannot contribute directly to the gluon transversity, it could be a good observable to find a new non-nucleonic component beyond the simple bound system of nucleons in nuclei.
\end{abstract}

DOI: 10.1103/PhysRevD.101.054011

\section{INTRODUCTION}

Although the nucleon spin is one of fundamental physics quantities, its origin is not understood yet. It used to be interpreted by a combination of three spin- $1 / 2$ quarks according to the basic quark model [1]. Namely, if two quark spins are aligned to the nucleon spin and the other quark spin is opposite, the nucleon spin should be understood. This simple description had been taken as granted

\footnotetext{
*shunzo.kumano@kek.jp

qintao@post.kek.jp
}

Published by the American Physical Society under the terms of the Creative Commons Attribution 4.0 International license. Further distribution of this work must maintain attribution to the author(s) and the published article's title, journal citation, and DOI. Funded by SCOAP . for a long time until the European Muon Collaboration (EMC) experiment found that this picture is basically wrong in 1988 by showing that the contribution from the quark spin is a small fraction [2]. We now know that gluon-spin and partonic orbital-angular-momentum contributions could be significant as sources of the nucleon spin.

Since the EMC discovery, studies on high-energy polarized-hadron reactions have been done to clarify the origin of the nucleon spin, mainly through longitudinally polarized structure functions [3]. In addition, there had been discussions how to decompose the nucleon spin into quark- and gluon-spin components and orbital-angular-momentum contributions in a gauge invariant way [4]. There are also studies in lattice QCD on the spin decomposition [5]. Furthermore, efforts have been made recently to obtain $x$-dependent parton distributions from lattice QCD [6]. Now, experimental clarifications on gluon-spin 
and partonic orbital-angular-momentum contributions become necessary. For probing the orbital-angularmomentum part, we need to investigate three-dimensional structure functions [7], namely, generalized parton distributions (GPDs) [8], generalized distribution amplitudes [9], and transverse-momentum-dependent parton distributions (TMDs) [10]. Such experimental studies are under investigations at experimental facilities in the world [11].

In spite of much progress on longitudinal spin physics, the transversely polarized structure functions are not known well [12-17], although there are some recent studies on quark transversity distributions [18]. Such studies provide an important and alternative information in solving the nucleon spin puzzle. In particular, the quark transversity distributions of the nucleon are decoupled from the gluon transversity in the $Q^{2}$ evolution $[19,20]$ due to the helicityflip (chiral-odd) property, which is an important difference from the longitudinal spin. Therefore, studies of the transversity distributions are other tests of our understanding on nucleon spin by different observables. In addition, since electric dipole moments of hadrons, such as the neutron, are proportional to the transversity distributions [21], the transversity studies are valuable also for searching physics beyond the standard model by measuring the electric dipole moments. There are also transversity GPD studies [22].

For understanding of transverse-polarization physics, the gluon transversity distribution should be investigated in addition to the quark transversity. The gluon transversity is not experimentally measured at this stage, whereas we have a rough idea on the quark transversity distributions [18]. However, there are future experimental projects to measure them accurately at Thomas Jefferson National Accelerator Facility (JLab) and Electron-Ion Collider (EIC) [23,24]. Therefore, much progress is expected for the gluon transversity in the near future because of the JLab experiment on the gluon transversity with the polarized-deuteron target [25].

On the other hand, independent experiments are desirable at other experimental facilities, especially at hadron accelerator facilities, to probe different kinematical regions of the gluon transversity from the JLab one. In particular, the large $Q^{2}$ region $\left(M_{J / \psi}^{2}<Q^{2}<M_{\Upsilon}^{2}\right)$ should be measured by the Drell-Yan process, in comparison with the JLab $Q^{2}$ region typically from a few $\mathrm{GeV}^{2}$ to several $\mathrm{GeV}^{2}$. The Fermilab spin-physics project SpinQuest is under preparation as the E1039 experiment [26], and the proton-deuteron Drell-Yan will be also possible with the polarized deuteron target.

The purpose of this work is to propose a new process to measure the gluon transversity for the first time at hadron facilities. Especially, we propose that the gluon transversity should be measured in the proton-deuteron Drell-Yan process by considering the Fermilab-E1039 experimental project. However, our formalism can be used in principle for the Drell-Yan experiments at any high-energy hadron accelerator facilities.
We may remind the reader that a hadron with spin at least one is necessary for studying the gluon transversity, which does not exist for the spin- $1 / 2$ nucleon, because the change of two units of spin $(\Delta s=2)$ is necessary for the gluon transversity [17]. The purpose of our work is to propose a possible process to probe the gluon transversity at hadron accelerator facilities as an alternative and independent method from the lepton scattering measurement at JLab and EIC.

Apart from the spin physics, the gluon transversity is a theoretically interesting quantity to probe an exotic aspect of the deuteron, hadrons, and nuclei. For example, the deuteron is a weak-bound state of a proton and a neutron mainly in the $\mathrm{S}$ wave with a small probability of D-state admixture. Since the internal nucleons do not contribute directly to the gluon transversity due to the spin- $1 / 2$ nature, the gluon transversity of the deuteron is expected to be a small quantity. However, if a finite distribution is found in future, it could indicate an existence of a non-nucleonic component or some other exotic hadronic mechanism within the deuteron. There are theoretical-model [27] and lattice-QCD [28] studies on this topic. For example, a contribution from the $\Delta \Delta$ component in the deuteron was estimated in Ref. [27] as a possible finite gluon transversity in the deuteron. Therefore, the gluon transversity distributions are interesting quantities, which shed light on unknown exotic aspects in the deuteron and nuclei beyond the simple bound systems of protons and neutrons.

In addition to the transversity, there are related studies on polarized deuteron structure functions. For example, the tensor-polarized structure function $b_{1}$ will be measured in the near future at JLab [29-31], and polarized protondeuteron reactions [32] could be investigated at Fermilab for measuring tensor-polarized distribution functions $[26,33]$. Since the conventional deuteron model cannot explain existing experimental measurements by the HERMES Collaboration [34], a new hadronic mechanism would be needed for their interpretation [35]. Such new aspects of the deuteron at high energies may be related to the gluon transversity distributions because they probe non-nucleonic component in the deuteron.

In this paper, the transversity distributions are explained by starting from the basic Pauli-Lubanski operator and matrix-element forms of local quark current operators in comparison with the longitudinally polarized parton distribution functions (PDFs) in Sec. II. Since the quark transversity distributions are directly related to electric dipole moments of hadrons, the relation is briefly explained. Then, the gluon transversity is explained. It exists in hadrons only with spin larger than or equal to one due to the helicity conservation. Next, the theoretical formalism is shown for the proton $(p)$-deuteron $(d)$ Drell-Yan process $p+d \rightarrow \mu^{+} \mu^{+}+X$ in Sec. III, including kinematical variables, polarizations of spin-1 deuteron, hadron correlation functions in terms of the PDFs, and 
expression of the cross section $p+d \rightarrow \mu^{+} \mu^{+}+X$. Then, partonic matrix elements and actual cross sections are obtained. The proton-deuteron Drell-Yan cross sections are numerically shown in Sec. IV, and our study is summarized in Sec. V.

\section{TRANSVERSITY DISTRIBUTIONS}

\section{A. Pauli-Lubanski operator in Poincaré group and quark transversity distributions}

The transversity is not a popular terminology outside the high-energy spin-physics community, and the transverse spin and polarization are somewhat confusing as shown in Eqs. (8) and (9), so that its basics are first explained [17].

The four-dimensional space-time coordinate transformation $x^{\prime \mu}=\Lambda^{\mu}{ }_{\nu} x^{\nu}+a^{\mu}$ is called the inhomogeneous Lorentz transformation or the Poincare transformation. The invariance under this transformation is a fundamental symmetry in quantum field theory. Representations of the Poincaré group are classified by Casimir operators $p^{2}$ and $W^{2}$ [36]. Here, $p^{\mu}$ is the momentum operator which is the generator of translations, and $W^{\mu}$ is the Pauli-Lubanski operator which is the generator of Lorentz transformations. The Pauli-Lubanski operator is defined by the angular-momentum operator $J^{\nu \rho}$ and the momentum as

$$
W_{\mu}=\frac{1}{2} \varepsilon_{\mu \nu \rho \sigma} J^{\nu \rho} p^{\sigma},
$$

with the antisymmetric tensor definition $\varepsilon_{0123}=+1$. The angular-momentum operator is given by

$$
J^{\mu \nu}=\frac{1}{2} \sigma^{\mu \nu}+\left(x^{\mu} p^{\nu}-x^{\nu} p^{\mu}\right) .
$$

Here, the antisymmetric tensor $\sigma^{\mu \nu}$ is defined by $\sigma^{\mu \nu}=$ $\frac{i}{2}\left(\gamma^{\mu} \gamma^{\nu}-\gamma^{\nu} \gamma^{\mu}\right)$. The eigenvalues of $p^{2}$ and $W^{2}$ are $M_{N}^{2}$ and $-M_{N}^{2} s(s+1)$, respectively,

$p^{2}|p s\rangle=M_{N}^{2}|p s\rangle, \quad W^{2}|p s\rangle=-M_{N}^{2} s(s+1)|p s\rangle$,

where $M_{N}$ and $s(=1 / 2)$ are mass and spin of the nucleon.

From the Pauli-Lubanski operator, the polarization operator $\Pi$ could be defined for the nucleon as [17]

$$
\Pi \equiv-\frac{1}{M_{N}} W \cdot s=\frac{1}{2 M_{N}} \gamma_{5} \phi \not p=\frac{1}{2 M_{N} i} \gamma_{5} \sigma_{\mu \nu} s^{\mu} p^{\nu},
$$

where it is expressed by the spin and momentum vectors. The spin vector $s^{\mu}$ satisfies $s^{2}=-1$ and $s \cdot p=0$, and it is generally expressed as

$$
s^{\mu}=\left(\frac{\vec{p} \cdot \hat{n}}{M_{N}}, \hat{n}+\frac{\vec{p} \cdot \hat{n}}{M_{N}\left(M_{N}+p^{0}\right)} \vec{p}\right),
$$

where $\hat{n}$ is a unit vector in three-dimensional space to indicate the spin-polarization direction. The longitudinal polarization is given by $\hat{n}= \pm \vec{p} /|\vec{p}|$, and the transverse one is by $\hat{n}=\hat{n}_{\perp}$ where $\hat{n}_{\perp}$ is a two-dimensional transverse unit vector. The polarization vector of Eq. (4) becomes the helicity operator,

$$
\Pi_{\|}=\frac{1}{2} \frac{\vec{\Sigma} \cdot \vec{p}}{|\vec{p}|}=\frac{1}{2} \Sigma_{\|}=\frac{1}{2} \gamma_{5} \gamma_{0} \gamma_{\|}=\frac{\sigma_{\|}}{2}\left(\begin{array}{ll}
I & 0 \\
0 & I
\end{array}\right),
$$

if the nucleon is longitudinally polarized. Here, the longitudinal direction is taken along the third axis $\left(|\vec{p}|=p_{\|}=p_{3}\right)$. The spin operator $\vec{\Sigma}$ is defined by

$$
\vec{\Sigma}=\gamma_{5} \gamma_{0} \vec{\gamma}
$$

$\sigma_{\|}$is the longitudinal Pauli-spin matrix defined by $\sigma_{\|} \equiv$ $\vec{\sigma} \cdot \vec{p} /|\vec{p}|$, and $I$ is the $2 \times 2$ identity matrix. This helicity operator commutes with the free-quark Hamiltonian $H_{0}=\alpha_{3} p_{3}=\gamma_{0} \gamma_{3} p_{3}$, so that it is a conserved quantity.

On the other hand, if the nucleon is transversely polarized, the transverse polarization and spin operators may be given, from Eqs. (4) and (7), by

$$
\Pi_{\perp}=\frac{1}{2 M_{N}} \gamma_{5} \phi \perp p, \quad \Sigma_{\perp}=\gamma_{5} \gamma_{0} \gamma_{\perp},
$$

where $s_{\perp}^{\mu}=\left(0, \hat{n}_{\perp}\right) \equiv n_{\perp}^{\mu}$, and $\Sigma_{\perp}$ and $\gamma_{\perp}$ are defined by $\Sigma_{\perp}=\hat{n}_{\perp} \cdot \vec{\Sigma}$ and $\gamma_{\perp}=\hat{n}_{\perp} \cdot \vec{\gamma}=\gamma_{1} \cos \phi_{\perp}+\gamma_{2} \sin \phi_{\perp}$ with the azimuthal angle $\phi_{\perp}$ of $\hat{n}_{\perp}$. However, they do not commute with the free Hamiltonian. It means that there are no eigenstates of $\Pi_{\perp}$ or $\vec{\Sigma}_{\perp}$ with $H_{0}$, so that the quarks in the transversely polarized nucleon cannot have a definite transverse-spin state with the polarization or spin operator of Eq. (8). However, if the transverse-polarization operator $\tilde{\Pi}_{\perp}$ is defined with an extra $\gamma_{0}$ with $\Sigma_{\perp}$ by

$$
\tilde{\Pi}_{\perp} \equiv \frac{1}{2} \gamma_{0} \Sigma_{\perp}=\frac{1}{2} \gamma_{5} n_{\perp} \cdot \gamma=\frac{\sigma_{\perp}}{2}\left(\begin{array}{cc}
I & 0 \\
0 & -I
\end{array}\right),
$$

it commutes with $H_{0}$. Here, the transverse Pauli spin matrix is defined by $\sigma_{\perp}=\hat{n}_{\perp} \cdot \vec{\sigma}$. We call this polarization as "transversity." It means that the quarks exist in the nucleon as a definite transverse-polarization state for $\tilde{\Pi}_{\perp}$, although the spin eigenstate does not exist for the operators $\Pi_{\perp}$ and $\vec{\Sigma}_{\perp}$. The transversity distributions are denoted $\Delta_{T} q$ for quarks and $\Delta_{T} g$ for gluon throughout this paper. However, there are other notations $h_{T}$ [12], $\Delta_{1} q$ [13], $h_{1}$ [14], and $\delta q$ [15] for quarks and other ones $\left(\Delta_{2} G, a, \Delta_{L} g, \delta G, h_{1 \mathrm{TT}, g}\right.$, $\Delta_{T} g$ ), as shown later in Eq. (68), for gluon, so that one may pay attention to the notation in reading past papers on the transversity.

Since the transverse spin $s_{\perp}$ was mentioned for the nucleon, it is briefly explained in the following. The polarized charged-lepton deep inelastic scattering (DIS) from a polarized nucleon is described by the antisymmetric 
hadron tensor in terms of two polarized structure functions $g_{1}$ and $g_{2}$,

$$
\begin{aligned}
W_{\mu \nu}^{(A)} & =\frac{2 M_{N}}{p \cdot q} \varepsilon_{\mu \nu \alpha \beta} q^{\alpha}\left[s^{\beta} g_{1}+\left(s^{\beta}-\frac{s \cdot q}{p \cdot q} p^{\beta}\right) g_{2}\right] \\
& =\frac{2 M_{N}}{p \cdot q} \varepsilon_{\mu \nu \alpha \beta} q^{\alpha}\left(s_{\|}^{\beta} g_{1}+s_{\perp}^{\beta} g_{T}\right),
\end{aligned}
$$

where $g_{T}$ is defined by

$$
g_{T} \equiv g_{1}+g_{2} .
$$

The nucleon spin vector is decomposed into the longitudinal and transverse ones as

$$
s^{\mu}=s_{\|}^{\mu}+s_{\perp}^{\mu}=\frac{\lambda_{N}}{M_{N}} p^{\mu}+s_{\perp}^{\mu},
$$

where $\lambda_{N}$ is the nucleon helicity. It indicates that the transverse-spin $s_{\perp}^{\mu}$ contribution to the cross section is suppressed by the factor $M_{N} / p^{+}$, where $p^{+}$is the lightcone momentum $p^{+}=\left(p^{0}+p^{3}\right) / \sqrt{2}$, in comparison with the longitudinal term. As explained, the transverse spin is not a conserved quantity, the structure function $g_{T}$ does not allow a simple probabilistic interpretation of the leading-twist level, although it can be measured experimentally in the standard polarized charged-lepton scattering measurement.

Therefore, the transverse-polarization physics is investigated by the transversity distributions in the twist-2-level collinear framework. Experimentally, the quark transversity distributions are determined, for example, by analyzing the data of semi-inclusive DIS process and proton-proton collisions with dihadron production [18]. There are other possibilities such as semi-inclusive hadron-production processes and Drell-Yan processes for the quark transversity distribution. However, there is little information on how to determine the gluon transversity at this stage. Especially, there is no paper to investigate the gluon transversity by using hadron experimental facilities as far as we are aware. Here, a possible process is proposed in this work.

\section{B. Longitudinally polarized and transversity distributions for quarks}

First, we explain quark transversity distributions. The longitudinally polarized quark distribution functions are given by the difference between the quark distributions with spin parallel to the nucleon spin and the ones with antiparallel spin: $\Delta q(x)=q_{+}(x)-q_{-}(x)$, where $x$ is the momentum fraction carried by a quark, as illustrated in Fig. 1(a). Here, + and - indicate parallel and antiparallel quark spins to the longitudinal nucleon spin. They are relatively well determined now for the nucleon by polarized lepton DIS and polarized proton-proton collisions. For the transversely polarized nucleon, similar distributions called
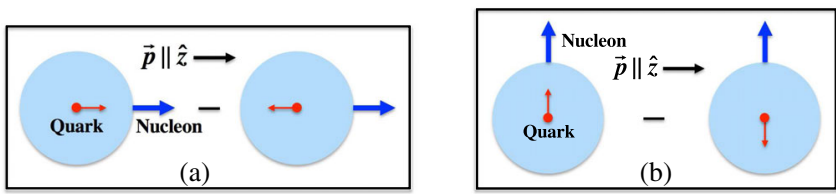

FIG. 1. Illustration of (a) longitudinally polarized quark distribution and (b) quark transversity distribution.

transversity distributions are expressed as $\Delta_{T} q(x)=$ $q_{\uparrow}(x)-q_{\downarrow}(x)$, where $\uparrow$ and $\downarrow$ indicate parallel and antiparallel quark polarizations, as defined by the polarization operator of Eq. (9), to the transversely polarized nucleon spin, as illustrated in Fig. 1(b).

The unpolarized, longitudinally polarized, and transversity distribution functions are defined for quarks by the following matrix elements [17]:

$$
\begin{aligned}
q(x)= & \int \frac{d \xi^{-}}{4 \pi} e^{i x p^{+} \xi^{-}}\left\langle p\left|\bar{\psi}(0) \gamma^{+} \psi(\xi)\right| p\right\rangle_{\xi^{+}=\vec{\xi}_{\perp}=0}, \\
\Delta q(x)= & \int \frac{d \xi^{-}}{4 \pi} e^{i x p^{+} \xi^{-}}\left\langle p s_{L}\left|\bar{\psi}(0) \gamma^{+} \gamma_{5} \psi(\xi)\right| p s_{L}\right\rangle_{\xi^{+}=\vec{\xi}_{\perp}=0}, \\
\Delta_{T} q(x)= & \int \frac{d \xi^{-}}{4 \pi} e^{i x p^{+} \xi^{-}} \\
& \times\left\langle p s_{T j}\left|\bar{\psi}(0) i \gamma_{5} \sigma^{j+} \psi(\xi)\right| p s_{T j}\right\rangle_{\xi^{+}=\vec{\xi}_{\perp}=0}
\end{aligned}
$$

where $s_{L}$ and $s_{T j}(j=1$ or 2$)$ indicate longitudinal and transverse polarizations of the nucleon, and $\psi$ is the quark field. Here, gauge links for satisfying the color gauge invariance are abbreviated because they do not play an important role in the collinear PDFs. These distribution functions are leading twist (twist-2) ones. In Sec. II A, we introduced the structure function $g_{T}$ associated with the transverse spin. It is also written in an operator matrix element in the similar way as

$$
\begin{aligned}
g_{T, q}(x)= & \frac{p^{+}}{M_{N}} \int \frac{d \xi^{-}}{4 \pi} e^{i x p^{+} \xi^{-}} \\
& \times\left\langle p s_{T}\left|\bar{\psi}(0) \gamma_{\perp} \gamma_{5} \psi(\xi)\right| p s_{T}\right\rangle_{\xi^{+}=\vec{\xi}_{\perp}=0} .
\end{aligned}
$$

This is a twist-3 structure function, which is suppressed typically by the kinematical factor of the order of $M_{N} / p^{+}$ in cross sections. By defining "good" $(+)$ and "bad" (-) components of the field $\psi$ as $\psi=\psi_{+}+\psi_{-}, \psi_{ \pm} \equiv$ $\left(\gamma^{\mp} \gamma^{ \pm} / 2\right) \psi$ [14], the operator of the longitudinally polarized quark distributions $\Delta q(x)$ in Eq. (13) is expressed by $\bar{\psi} \gamma^{+} \gamma_{5} \psi=\bar{\psi}_{+} \gamma^{+} \gamma_{5} \psi_{+}$, namely only by the good components. For the distribution $g_{T, q}(x)$, it is given only by the cross combination of $\psi_{+}$and $\psi_{-}$as $\bar{\psi} \gamma_{\perp} \gamma_{5} \psi=$ $\bar{\psi}_{+} \gamma_{\perp} \gamma_{5} \psi_{-}+\bar{\psi}_{-} \gamma_{\perp} \gamma_{5} \psi_{+}$, which indicates that it is a twist-3 distribution.

As known in the DIS formalism, structure functions of the nucleon are given by the imaginary part of forward scattering amplitudes by the optical theorem. The PDFs are 


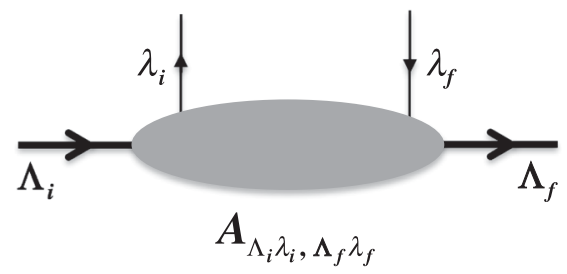

FIG. 2. Parton-hadron forward scattering amplitude $A_{\Lambda_{i} \lambda_{i}, \Lambda_{f} \lambda_{f}}$ with the hadron helicities $\Lambda_{i}$ and $\Lambda_{f}$ and parton ones $\lambda_{i}$ and $\lambda_{f}$.

expressed by parton-hadron forward scattering amplitudes illustrated in Fig. 2. The amplitude is denoted as $A_{\Lambda_{i} \lambda_{i}, \Lambda_{f} \lambda_{f}}$ with the initial and final hadron helicities $\Lambda_{i}$ and $\Lambda_{f}$ and parton ones $\lambda_{i}$ and $\lambda_{f}$. The helicity conservation indicates the relation [37]

$$
\Lambda_{i}-\lambda_{i}=\Lambda_{f}-\lambda_{f}
$$

where the minus signs exist for the parton helicities due to outgoing and incoming particles in comparison with the hadron helicities. The PDFs are related to the helicity amplitudes as $[17,37]$

$$
\begin{aligned}
q(x) & =q_{+}(x)+q_{-}(x) \sim \operatorname{Im}\left(A_{++,++}+A_{+-,+-}\right), \\
\Delta q(x) & =q_{+}(x)-q_{-}(x) \sim \operatorname{Im}\left(A_{++,++}-A_{+-,+-}\right), \\
\Delta_{T} q(x) & =q_{\uparrow}(x)-q_{\downarrow}(x) \sim \operatorname{Im} A_{++,--} .
\end{aligned}
$$

The last relation $\Delta_{T} q \sim \operatorname{Im} A_{++,--}$is given in Ref. [37]. It should be noted that the helicity amplitudes $A_{\Lambda_{i} \lambda_{i}, \Lambda_{f} \lambda_{f}}$ are used in this paper and in Ref. [37]. However, one needs to be careful about a notation difference in some other papers, because the amplitudes are often defined by spin components along the quantization axis as given in Ref. [30]. For example, the relation is $\Delta_{T} q \sim \operatorname{Im} A_{-+,+-}$in the $A_{h H, h^{\prime} H^{\prime}-}$ notation [25,28], where $h$ and $H\left(h^{\prime}\right.$ and $\left.H^{\prime}\right)$ are initial (final) quark and hadron spin components.

If the spin states are defined by the transversity basis $|\uparrow\rangle$ and $|\downarrow\rangle$, they are expressed by the longitudinally polarized states as [17]

$$
\begin{aligned}
& |\uparrow\rangle=\frac{1}{\sqrt{2}}[|+\rangle+|-\rangle], \\
& |\downarrow\rangle=\frac{1}{\sqrt{2}}[|+\rangle-|-\rangle],
\end{aligned}
$$

where the direction of the polarization $\uparrow$ is taken along the $x$ axis. Therefore, if the amplitudes are defined by the transversely polarized states, the transversity distribution is given by

$$
\Delta_{T} q(x)=q_{\uparrow}(x)-q_{\downarrow}(x) \sim \operatorname{Im}\left(A_{\uparrow \uparrow, \uparrow \uparrow}-A_{\uparrow \downarrow, \uparrow \downarrow}\right) .
$$

The transversity distributions are important leading-twist functions for clarifying the internal structure of the nucleon.

\section{Electric dipole moment of neutron}

The transversity is an important physics quantity not only for clarifying the nature and origin of the nucleon spin but also for finding a signature of beyond the standard model by observing electric dipole moments of the neutron and other hadrons. Therefore, its connection to the electric dipole moment is briefly explained. The neutron electromagnetic current is expressed as [21]

$$
\begin{aligned}
\left\langle n\left|J_{\mu}^{\mathrm{em}}\right| n\right\rangle= & \bar{u}\left(p^{\prime}\right)\left[\gamma_{\mu} F_{1}\left(q^{2}\right)+\frac{\kappa}{2 M_{N}} i \sigma_{\mu \nu} q^{\nu} F_{2}\left(q^{2}\right)\right. \\
& \left.+\frac{d_{n}}{2 M_{N}} \gamma_{5} \sigma_{\mu \nu} q^{\nu} F_{3}\left(q^{2}\right)\right] u(p),
\end{aligned}
$$

by including the time-reversal odd term with the form factor $F_{3}$ in addition to the ordinary parity and timereversal even terms with the form factors $F_{1}$ and $F_{2}$. Here, $\kappa$ is the anomalous magnetic moment, $F_{1}$ and $F_{2}$ are Dirac and Pauli form factors, and they are related to the electric and magnetic form factors as $G_{E}\left(q^{2}\right)=F_{1}\left(q^{2}\right)+$ $q^{2} /\left(2 M_{N}^{2}\right) \kappa F_{2}\left(q^{2}\right)$ and $G_{M}\left(q^{2}\right)=F_{1}\left(q^{2}\right)+\kappa F_{2}\left(q^{2}\right)$. The initial and final neutron momenta are denoted as $p$ and $p^{\prime}, q$ is the momentum transfer given by $q=p-p^{\prime}$, and $u(p)$ is the Dirac spinor for the neutron. The last $F_{3}$ term is the time-reversal odd one, in combination with the electromagnetic field $A^{\mu}$ for the Hamiltonian [21], with the factor of the neutron electric dipole moment (EDM) $d_{n}$ in the unit of $e /\left(2 M_{N}\right)$. The electric dipole form factor $F_{3}$ is normalized as $F_{3}(0)=1$ at $q^{2}=0$. Here, we use the function notation $F_{3}$ which has been used so far in EDM studies; however, the function $F_{3}$ is conventionally used for the structure function $F_{3}$ in neutrino scattering. They should not be confused.

On the other hand, the neutron EDM is expressed by integrals of the transversity distributions, so-called the tensor charge $\Delta_{T} q$, as [21]

$$
\begin{aligned}
d_{n} & =\sum_{q} d_{q} \Delta_{T} q, \\
\Delta_{T} q & \equiv \int_{0}^{1} d x\left[\Delta_{T} q(x)-\Delta_{T} \bar{q}(x)\right],
\end{aligned}
$$

where $d_{q}$ is the quark EDM. Namely, the neutron EDM is investigated theoretically by calculating the quark EDMs in the standard model or some models beyond the standard model, and they should be multiplied by the tensor charge in order to compare with experimental measurements. Therefore, the studies of transversity distributions have impact on investigations of physics beyond the standard model by observing the EDMs of the neutron, other hadrons, and nuclei. 


\section{Gluon transversity in hadrons with spin $\geq 1$}

As shown in Eq. (16), the transversity distribution $\Delta_{T} q(x)$ is associated with the quark spin flip $\left(\lambda_{i}=+\right.$, $\lambda_{f}=-$ ), so that it is a chiral-odd distribution. The quark transversity exists in the nucleon because the spin flip $\Delta s=$ 1 is possible in the spin- $1 / 2$ nucleon, whereas the gluon transversity $\Delta_{T} g$ does not exist in the nucleon because the spin flip $\Delta s=2$ is not possible. Therefore, the quark transversity distributions evolve in the scale $Q^{2}$ without the corresponding gluon distribution in the nucleon $[19,20]$. This situation is very different from the longitudinally polarized PDFs, where the quark and gluon distributions couple with each other in the $Q^{2}$ evolution as we usually have in the unpolarized PDFs. This is an important test of perturbative QCD in high-energy spin physics.

In the same way with the quark transversity expression of Eq. (18), the gluon transversity is written by the helicity distribution as $[25,28]$

$$
\Delta_{T} g(x) \sim \operatorname{Im} A_{++,--} .
$$

This equation indicates that the spin flip of two units $\Delta s=$ $2\left(\left|\lambda_{f}-\lambda_{i}\right|=\left|\Lambda_{f}-\Lambda_{i}\right|=2\right)$ is necessary for the gluon transversity $\Delta_{T} g$, and it is illustrated in Fig. 3. In order to find the gluon transversity, hadrons with spin $\geq 1$ should be used. The most simple and stable spin-1 hadron or nucleus is the deuteron, so that it is used first for future experimental studies of the gluon transversity. There is an experimental proposal to measure it in the polarized electron-deuteron DIS by looking at the azimuthal angle of the deuteron-spin polarization [25]. In our work, we investigate a possibility to investigate the gluon transversity in the deuteron by hadron accelerator facilities as an alternative way to the lepton-facility measurements.

In the similar way with Eq. (13), the gluon transversity distribution is written in the matrix element form,

$$
\begin{aligned}
\Delta_{T} g(x)= & \varepsilon_{\mathrm{TT}, \alpha \beta} \int \frac{d \xi^{-}}{2 \pi} x p^{+} e^{i x p^{+} \xi^{-}} \\
& \times\left\langle p E_{x}\left|A^{\alpha}(0) A^{\beta}(\xi)\right| p E_{x}\right\rangle_{\xi^{+}=\vec{\xi}_{\perp}=0},
\end{aligned}
$$

where $\varepsilon_{\mathrm{TT}}^{\alpha \beta}=+1$ for $\alpha=\beta=1, \varepsilon_{\mathrm{TT}}^{\alpha \beta}=-1$ for $\alpha=\beta=2$, and the other components are zero, as defined later in

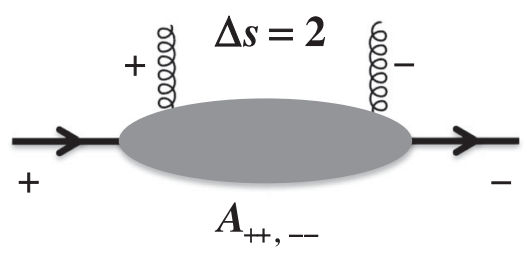

FIG. 3. Gluon-hadron forward scattering amplitude $A_{++,--}$ with the spin flip of $2(\Delta s=2)$ for finding the gluon transversity. The hadron spin should be $s \geq 1$. For example, it is spin-1 deuteron.
Eq. (39). The notation $E_{x}$ indicates the linear polarization of the deuteron along the positive $x$-axis. This expression for $\Delta_{T} g(x)$ is obtained by using the gluon correlation function of Eq. (58) and the collinear expression of Eq. (67). In this equation, $A^{\alpha}$ is the gluon field $A^{\alpha}=$ $A_{a}^{\alpha} t^{a}$ which includes the $\mathrm{SU}(3)$ generator $t^{a}$ expressed by the Gell-Mann matrix as $t^{a}=\lambda^{a} / 2$ with the color index $a$. Here, the summation is taken over $a$. Therefore, $\Delta_{T} g$ is given by the linear-polarization difference $\varepsilon_{x}-\varepsilon_{y}$ for the gluon in the linearly polarized $\left(E_{x}\right)$ deuteron. The linear polarizations for the deuteron $\left(E_{x}, E_{y}\right)$ and the gluon $\left(\varepsilon_{x}, \varepsilon_{y}\right)$ are explained in Secs. III A and III B. We should mention that the name "gluon transversity" is misleading in the sense that it does not mean the transverse polarization of the gluon but it is actually on the linear polarization.

\section{FORMALISM FOR DRELL-YAN PROCESS $p+d \rightarrow \mu^{+} \mu^{-}+X$}

Our formalism is explained for describing the Drell-Yan cross section $p+d \rightarrow \mu^{+} \mu^{-}+X$ in this section. Since this work is on deuteron spin physics, especially on gluon transversity, we introduce polarizations of spin-1 deuteron. Then, the cross section formalism is discussed.

\section{A. Spin-1 deuteron polarizations}

Since polarizations of the spin-1 deuteron, which contains tensor polarizations, are not familiar, they are explained in general by using the spin-density matrix. Let us consider a spin state $|\psi\rangle$ for a particle of spin $s$, and it is expanded by eigenstates of the $z$ component of the spin operator $s_{z}$ with the expansion coefficients $c_{m}$ as $|\psi\rangle=\sum_{m} c_{m}|s m\rangle$ [38]. Matrix elements of an operator $\hat{O}$ are denoted as $O_{m^{\prime} m}=\left\langle s m^{\prime}|\hat{O}| s m\right\rangle$, and then expectation value in the state $|\psi\rangle$ is expressed as $\langle\psi|\hat{O}| \psi\rangle=\sum_{m, m^{\prime}} c_{m^{\prime}}^{*} c_{m} O_{m^{\prime} m}$. If the state is an incoherent mixture of pure states $\left|\psi^{(i)}\right\rangle$ with the probability $p^{(i)}$, the expectation value is written as $\langle\hat{O}\rangle=\sum_{i} p^{(i)}\left\langle\psi^{(i)}|\hat{O}| \psi^{(i)}\right\rangle=$ $\sum_{m, m^{\prime}} O_{m^{\prime} m} \sum_{i} p^{(i)} c_{m^{\prime}}^{(i) *} c_{m}^{(i)}$. Defining the spin-density matrix $\rho_{m m^{\prime}}$ as

$$
\rho_{m m^{\prime}}=\sum_{i} p^{(i)} c_{m}^{(i)} c_{m^{\prime}}^{(i) *}
$$

we obtain

$$
\langle\hat{O}\rangle=\sum_{m, m^{\prime}} O_{m^{\prime} m} \rho_{m m^{\prime}}=\operatorname{Tr}(O \rho) .
$$

Therefore, if the spin-density matrix is known, the expectation value over the ensemble can be calculated for operators.

For example, the density matrix is given by $\boldsymbol{\rho}_{1 / 2}=$ $\left(1+s_{i} \boldsymbol{\sigma}_{i}\right) / 2$, where the summation is taken over $i=1,2$, and 3 , with the Pauli matrix $\boldsymbol{\sigma}_{i}$, and the spin-polarization 
vector is given by $s_{i}=\left\langle\boldsymbol{\sigma}_{i}\right\rangle=\operatorname{Tr}\left(\boldsymbol{\rho}_{1 / 2} \boldsymbol{\sigma}_{i}\right)$. In the similar way, the density matrix for a spin-1 particle is given in the Cartesian coordinates as [38-40]

$$
\boldsymbol{\rho}=\frac{1}{3}\left(1+\frac{3}{2} S_{i} \boldsymbol{\Sigma}_{i}+3 T_{i j} \boldsymbol{\Sigma}_{i j}\right)
$$

where $\boldsymbol{\Sigma}_{i}(i=1,2,3)$ are $3 \times 3$ spin matrices for the spin-1 deuteron,

$$
\begin{array}{ll}
\boldsymbol{\Sigma}_{x}=\frac{1}{\sqrt{2}}\left(\begin{array}{ccc}
0 & 1 & 0 \\
1 & 0 & 1 \\
0 & 1 & 0
\end{array}\right), \quad \boldsymbol{\Sigma}_{y}=\frac{i}{\sqrt{2}}\left(\begin{array}{ccc}
0 & -1 & 0 \\
1 & 0 & -1 \\
0 & 1 & 0
\end{array}\right), \\
\boldsymbol{\Sigma}_{z}=\left(\begin{array}{ccc}
1 & 0 & 0 \\
0 & 0 & 0 \\
0 & 0 & -1
\end{array}\right),
\end{array}
$$

and $\boldsymbol{\Sigma}_{i j}$ are spin tensors defined by

$$
\boldsymbol{\Sigma}_{i j}=\frac{1}{2}\left(\boldsymbol{\Sigma}_{i} \boldsymbol{\Sigma}_{j}+\boldsymbol{\Sigma}_{j} \boldsymbol{\Sigma}_{i}\right)-\frac{2}{3} \boldsymbol{I} \delta_{i j}
$$

Here, $\boldsymbol{I}$ is the $3 \times 3$ identity matrix, and the $\boldsymbol{\Sigma}_{i j}$ convention of Refs. [39,40] is used instead of the one in Ref. [38]. The spin polarization vector $S_{i}$ is given by

$$
S_{i}=\left\langle\boldsymbol{\Sigma}_{i}\right\rangle=\operatorname{Tr}\left(\boldsymbol{\rho} \boldsymbol{\Sigma}_{i}\right),
$$

and the tensor $T_{i j}$ is a real and traceless one given by

$$
T_{i j}=\left\langle\boldsymbol{\Sigma}_{i j}\right\rangle=\operatorname{Tr}\left(\boldsymbol{\rho} \boldsymbol{\Sigma}_{i j}\right) .
$$

The spin vector and tensor are parametrized in the rest frame of the deuteron as $[39,41,42]$

$$
\begin{aligned}
& \boldsymbol{S}=\left(S_{T}^{x}, S_{T}^{y}, S_{L}\right), \\
& \boldsymbol{T}=\frac{1}{2}\left(\begin{array}{ccc}
-\frac{2}{3} S_{L L}+S_{\mathrm{TT}}^{x x} & S_{\mathrm{TT}}^{x y} & S_{L T}^{x} \\
S_{\mathrm{TT}}^{x y} & -\frac{2}{3} S_{L L}-S_{\mathrm{TT}}^{x x} & S_{L T}^{y} \\
S_{L T}^{x} & S_{L T}^{y} & \frac{4}{3} S_{L L}
\end{array}\right) .
\end{aligned}
$$

We use the tensor $\boldsymbol{T}$ in Refs. [39,41,42], whereas the factor $-(2 / 3) S_{L L}$ is denoted as $S_{L L}$ in Ref. [40]. The spin vector and tensor are written in terms of the polarization vector $\vec{E}$ of the deuteron as

$$
\vec{S}=\operatorname{Im}\left(\vec{E}^{*} \times \vec{E}\right), \quad T_{i j}=\frac{1}{3} \delta_{i j}-\operatorname{Re}\left(E_{i}^{*} E_{j}\right),
$$

and their covariant forms are given by $[30,31,40]$

$$
\begin{aligned}
S^{\mu} & =\frac{1}{M} \varepsilon^{\mu \nu \alpha \beta} p_{\nu} \operatorname{Im}\left(E_{\alpha}^{*} E_{\beta}\right), \\
T^{\mu \nu} & =-\frac{1}{3}\left(g^{\mu \nu}-\frac{p^{\mu} p^{\nu}}{p^{2}}\right)-\operatorname{Re}\left(E^{\mu *} E^{\nu}\right) .
\end{aligned}
$$

Here, $M$ and $p$ are the deuteron mass and momentum. At this stage, the deuteron spin quantization axis is taken as the $z$ direction; however, $-z$ direction is taken later in calculating the cross section along the deuteron momentum direction. Then, the deuteron polarization vector $E$ and also gluon polarization vector $\varepsilon$ are defined as

$$
\begin{aligned}
& E_{ \pm}=\varepsilon_{ \pm}=\frac{1}{\sqrt{2}}(0, \mp 1,-i, 0), \quad E_{0}=\varepsilon_{0}=(0,0,0,1), \\
& E_{x}=\varepsilon_{x}=\frac{1}{\sqrt{2}}\left(\varepsilon_{-}-\varepsilon_{+}\right)=(0,1,0,0), \\
& E_{y}=\varepsilon_{y}=\frac{i}{\sqrt{2}}\left(\varepsilon_{-}+\varepsilon_{+}\right)=(0,0,1,0) .
\end{aligned}
$$

Using these quantities, we express the spin-density matrix of Eq. (25) as [39,41,42]

$$
\boldsymbol{\rho}=\left(\begin{array}{ccc}
\frac{1}{3}+\frac{S_{L}}{2}+\frac{S_{L L}}{3} & \frac{S_{T}^{x}-i S_{T}^{y}}{2 \sqrt{2}}+\frac{S_{L T}^{x}-i S_{L T}^{y}}{2 \sqrt{2}} & \frac{S_{T T}^{x x}-i S_{T T}^{x y}}{2} \\
\frac{S_{T}^{x}+i S_{T}^{y}}{2 \sqrt{2}}+\frac{S_{L T}^{x}+i S_{L T}^{y}}{2 \sqrt{2}} & \frac{1}{3}-\frac{2 S_{L L}}{3} & \frac{S_{T}^{x}-i S_{T}^{y}}{2 \sqrt{2}}-\frac{S_{L T}^{x}-i S_{L T}^{y}}{2 \sqrt{2}} \\
\frac{S_{T T}^{x x}+i S_{T T}^{x y}}{2} & \frac{S_{T}^{x}+i S_{T}^{y}}{2 \sqrt{2}}-\frac{S_{L T}^{x}+i S_{L T}^{y}}{2 \sqrt{2}} & \frac{1}{3}-\frac{S_{L}}{2}+\frac{S_{L L}}{3}
\end{array}\right) .
$$

The covariant forms of $S^{\mu}$ and $T^{\mu \nu}$ are generally expressed by the longitudinal and transverse polarizations as $[39,41,42]$

$$
\begin{aligned}
S^{\mu}= & S_{L} \frac{p^{+}}{M} \bar{n}^{\mu}-S_{L} \frac{M}{2 p^{+}} n^{\mu}+S_{T}^{\mu}, \\
T^{\mu \nu}= & \frac{1}{2}\left[\frac{4}{3} S_{L L} \frac{\left(p^{+}\right)^{2}}{M^{2}} \bar{n}^{\mu} \bar{n}^{\nu}+\frac{p^{+}}{M} \bar{n}^{\{\mu} S_{L T}^{\nu\}}\right. \\
& -\frac{2}{3} S_{L L}\left(\bar{n}^{\{\mu} n^{\nu\}}-g_{T}^{\mu \nu}\right)+S_{\mathrm{TT}}^{\mu \nu}-\frac{M}{2 p^{+}} n^{\{\mu} S_{L T}^{\nu\}} \\
& \left.+\frac{1}{3} S_{L L} \frac{M^{2}}{\left(p^{+}\right)^{2}} n^{\mu} n^{\nu}\right],
\end{aligned}
$$

where $a^{\{\mu} b^{\nu\}}$ indicates the symmetrization of the superscript indices $a^{\{\mu} b^{\nu\}} \equiv a^{\mu} b^{\nu}+a^{\nu} b^{\mu}$, and $g_{T}^{\alpha \beta}$ is defined by $g_{T}^{\alpha \beta}=g^{\alpha \beta}-\bar{n}^{\{\alpha} n^{\beta\}} \quad\left(g_{T}^{11}=g_{T}^{22}=-1, \quad\right.$ others $\left.=0\right) . \quad$ The lightlike vectors $\bar{n}$ and $n$ are defined by

$$
\begin{aligned}
& \bar{n}^{\mu}=\frac{1}{\sqrt{2}}(1,0,0,+1)=\left[1,0, \overrightarrow{0}_{T}\right]_{\mathrm{LC}}, \\
& n^{\mu}=\frac{1}{\sqrt{2}}(1,0,0,-1)=\left[0,1, \overrightarrow{0}_{T}\right]_{\mathrm{LC}},
\end{aligned}
$$


where the lightcone (LC) notation $a^{\mu}=\left[a^{+}, a^{-}, \vec{a}_{T}\right]_{\mathrm{LC}}$ is used with $a^{ \pm}=\left(a^{0} \pm a^{3}\right) / \sqrt{2}$. Replacing $S_{L L}$ in Ref. [40] by $-(2 / 3) S_{L L}$ so as to agree with the convention of Refs. [39,41,42], we have [40]

$$
\begin{aligned}
S^{\mu} & =\left[S_{L} \frac{p^{+}}{M},-S_{L} \frac{M}{2 p^{+}}, S_{T}^{x}, S_{T}^{y}\right]_{\mathrm{LC}}, \\
T^{\mu \nu} & =\frac{1}{2}\left[\begin{array}{cccc}
\frac{4\left(P^{+}\right)^{2}}{3 M^{2}} S_{L L} & -\frac{2}{3} S_{L L} & \frac{p^{+}}{M} S_{L T}^{x} & \frac{p^{+}}{M} S_{L T}^{y} \\
-\frac{2}{3} S_{L L} & \frac{M^{2}}{3\left(p^{+}\right)^{2}} S_{L L} & -\frac{M}{2 P^{+}} S_{L T}^{x} & -\frac{M}{2 p^{+}} S_{L T}^{y} \\
\frac{p^{+}}{M} S_{L T}^{x} & -\frac{M}{2 p^{+}} S_{L T}^{x} & S_{\mathrm{TT}}^{x x}-\frac{2}{3} S_{L L} & S_{\mathrm{TT}}^{x y} \\
\frac{p^{+}}{M} S_{L T}^{y} & -\frac{M}{2 p^{+}} S_{L T}^{y} & S_{\mathrm{TT}}^{x y} & -S_{\mathrm{TT}}^{x x}-\frac{2}{3} S_{L L}
\end{array}\right]_{\mathrm{LC}} .
\end{aligned}
$$

The lightcone matrix notation means that the first component of column or line is $a^{+}$, the second is $a^{-}$, the third is the transverse coordinate $x$, and the fourth is the transverse $y$.

For investigating the gluon transversity distribution in the deuteron, the linear polarization of the spin-1 deuteron should be considered. The deuteron is linearly polarized if its polarization is $E_{x}, E_{y}$, or in between as illustrated in Fig. 4. In the expression of the general density matrix, the linear-polarization asymmetry $E_{x}-E_{y}$ corresponds to the spin asymmetry $S_{\mathrm{TT}}^{\alpha \beta}$ in Refs. [39-42]. Therefore, the linearpolarization asymmetry of the cross section is calculated by taking $\left[d \sigma\left(E_{x}\right)-d \sigma\left(E_{y}\right)\right] / 2$ with the polarization $S_{\mathrm{TT}}^{x x}$ and by taking other polarization as zero. Later, the factor of 2 is multiplied in expressing Eq. (88) because the spin asymmetry $d \sigma\left(E_{x}\right)-d \sigma\left(E_{y}\right)$ is used for expressing the cross section, instead of $\left[d \sigma\left(E_{x}\right)-d \sigma\left(E_{y}\right)\right] / 2$. As given in Eq. (35), the spin asymmetry $S_{\mathrm{TT}}^{\mu \nu}$ is obtained from the tensor $T^{\mu \nu}$, by terminating other spin asymmetries $S_{L L}=S_{L T}^{x}=S_{L T}^{y}=S_{\mathrm{TT}}^{x y}=0$. Then, using the matrix form of Eq. (37), we obtain

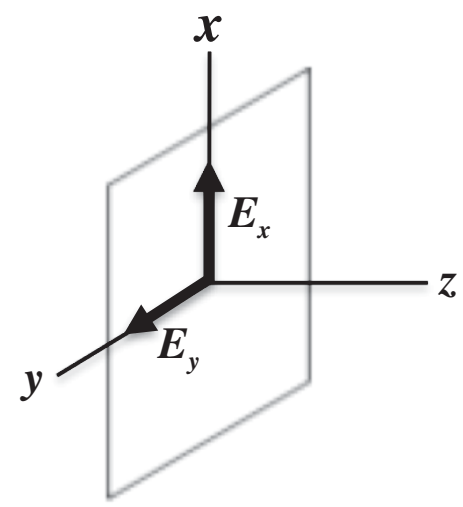

FIG. 4. Linear polarizations $E_{x}$ and $E_{y}$ of spin-1 deuteron.

$$
S_{\mathrm{TT}}^{\alpha \beta}=\left(\begin{array}{cccc}
0 & 0 & 0 & 0 \\
0 & -1 & 0 & 0 \\
0 & 0 & 1 & 0 \\
0 & 0 & 0 & 0
\end{array}\right),
$$

where $S_{\mathrm{TT}}^{x x}=-1$ is assigned for the linear-polarization asymmetry $E_{x}-E_{y}$, instead of $E_{y}-E_{x}$ in Refs. [39-42].

Next, we explain linear gluon polarizations which are related to the gluon transversity of the deuteron. As shown in Eq. (16), the transversity distribution is defined by the process with the gluon polarizations $\lambda_{i}=+1$ and $\lambda_{f}=-1$ or vice versa. Therefore, the gluon polarization tensor $\varepsilon_{\mathrm{TT}}^{\alpha \beta}$ is given by the polarization vectors defined in Eq. (33) as

$$
\varepsilon_{\mathrm{TT}}^{\alpha \beta} \equiv \varepsilon_{x}^{\alpha} \varepsilon_{x}^{* \beta}-\varepsilon_{y}^{\alpha} \varepsilon_{y}^{* \beta}=-\left(\varepsilon_{+}^{\alpha} \varepsilon_{-}^{* \beta}+\varepsilon_{-}^{\alpha} \varepsilon_{+}^{* \beta}\right) .
$$

This relation indicates the helicity flip of 2 in this process. For investigating the gluon transversity, the parent hadron, namely the deuteron in this work, should have spin larger than or equal to one. We notice that this gluon linearpolarization tensor $\varepsilon_{\mathrm{TT}}^{\alpha \beta}$ is the same as the linear-polarization asymmetry $S_{\mathrm{TT}}^{\alpha \beta}$ in Eq. (38) except for the sign

$$
\varepsilon_{\mathrm{TT}}^{\alpha \beta}=-S_{\mathrm{TT}}^{\alpha \beta} .
$$

The tensor $S_{\mathrm{TT}}^{\alpha \beta}$ (or $\varepsilon_{\mathrm{TT}}^{\alpha \beta}$ ) appears in calculating the Drell-Yan production cross section for finding the gluon transversity.

\section{B. Kinematical variables}

We express the cross section for the polarized protondeuteron Drell-Yan process $\left(p+d \rightarrow \mu^{+} \mu^{-}+X\right)$, which is illustrated in Fig. 5(a), in terms of the PDFs of the proton and the deuteron [43] including the gluon transversity distribution. The cross section for $A(p)+B(d) \rightarrow \mu^{+} \mu^{-}+X$ is described by the partonic subprocess $\sigma_{a b \rightarrow c d}$ in Fig. 5(b). The indices $A$ and $B$ indicate the proton $(p)$ and the deuteron $(d)$, and $c$ is used for the virtual photon $\left(\gamma^{*}\right)$.

Here, kinematical variables are explained for describing the process. First, the Mandelstam variables for the reaction $p+d \rightarrow \gamma^{*}+X$ are given by neglecting proton and deuteron masses as

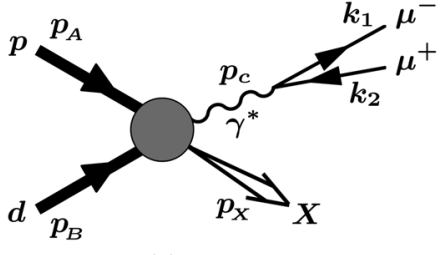

(a)

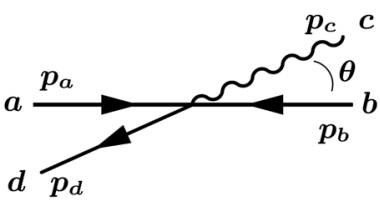

(b)
FIG. 5. (a) Proton-deuteron Drell-Yan process $p+d \rightarrow$ $\mu^{+} \mu^{-}+X$. (b) Parton reaction $a+b \rightarrow c+d$ in the center-ofmomentum frame. 
$s=\left(p_{A}+p_{B}\right)^{2}=\left(p_{c}+p_{X}\right)^{2}=2 p_{A} \cdot p_{B}$,

$t=\left(p_{A}-p_{c}\right)^{2}=\left(p_{B}-p_{X}\right)^{2}=Q^{2}-2 p_{A} \cdot p_{c}$,

$u=\left(p_{A}-p_{X}\right)^{2}=\left(p_{B}-p_{c}\right)^{2}=Q^{2}-2 p_{B} \cdot p_{c}$,

where $Q^{2}$ is defined by $Q^{2}=p_{c}^{2}$. It should be noted that the center-of-mass energy squared $s$ used in the Fermilab-E906 experimental proposal is different from our definition. Considering interactions with individual nucleons within the deuteron $[26,44]$, they defined $s$ as

$$
s_{\text {Fermilab-E906 }}=\left(p_{p}+\frac{p_{d}}{2}\right)^{2}=\frac{s}{2}
$$

for the proton-deuteron Drell-Yan process as written in their proposal. One needs to be careful about the definition difference in estimating the cross section numerically. This difference is also related to how to define scaling variables for partons in the deuteron, and the details of handling this difference are discussed in Sec. IV for showing numerical results.

In the proton-deuteron center-of-momentum (c.m.) frame, we denote the momenta as

$$
\begin{aligned}
p_{A} & =\frac{\sqrt{s}}{2}(1,0,0,1), \quad p_{B}=\frac{\sqrt{s}}{2}(1,0,0,-1), \\
q & \equiv p_{c}=\left(E, q_{T} \cos \phi, q_{T} \sin \phi, q_{L}\right), \\
p_{d} & =|\vec{q}|(1,-\sin \theta \cos \phi,-\sin \theta \sin \phi,-\cos \theta),
\end{aligned}
$$

where the polar and azimuthal angles of the virtual-photon momentum are given by $\theta$ and $\phi$, respectively, $|\vec{q}|$ is the photon momentum, and $q_{L}=|\vec{q}| \cos \theta$ and $q_{T}=|\vec{q}| \sin \theta$ are longitudinal and transverse momenta of photon. The $z$ direction is taken as the momentum direction for the proton $\left(z \| \vec{p}_{A}\right)$. The dimuon momentum is equal to the virtual photon momentum,

$$
q=k_{1}+k_{2},
$$

where $k_{1}$ and $k_{2}$ are $\mu^{-}$and $\mu^{+}$momenta, so that the scale $Q^{2}$ is the dimuon-mass squared,

$$
M_{\mu \mu}^{2}=\left(k_{1}+k_{2}\right)^{2}=Q^{2} .
$$

The dimensionless variable $\tau$ and the dimuon rapidity $y$ are defined by

$$
\tau=\frac{Q^{2}}{s}
$$

and

$$
y=\frac{1}{2} \ln \frac{E+q_{L}}{E-q_{L}}=-\ln [\tan (\theta / 2)]
$$

where $y$ is given by the energy $E$ and momentum $q_{L}$ in the c.m. frame. The photon momentum is also expressed by using the rapidity, the transverse momentum, and the transverse mass as

$$
q=\left(M_{T} \cosh y, q_{T} \cos \phi, q_{T} \sin \phi, M_{T} \sinh y\right),
$$

where the transverse mass is given by

$$
M_{T}=\sqrt{Q^{2}+\vec{q}_{T}^{2}} .
$$

In addition, the variables $x_{1}$ and $x_{2}$ are defined by [43]

$$
\begin{aligned}
& x_{1}=-\frac{u-Q^{2}}{s}=\frac{M_{T}}{\sqrt{s}} e^{y}, \\
& x_{2}=-\frac{t-Q^{2}}{s}=\frac{M_{T}}{\sqrt{s}} e^{-y}
\end{aligned}
$$

for describing the Drell-Yan cross section.

Next, kinematical variables are shown for parton reactions of Fig. 5(b). The initial parton momenta are given by the momentum fractions of partons, $x_{a}$ and $x_{b}$, with respect to their parent-hadron momenta as

$$
\begin{aligned}
& p_{a}=x_{a} P_{A}=x_{a} \frac{\sqrt{s}}{2}(1,0,0,1), \\
& p_{b}=x_{b} P_{B}=x_{b} \frac{\sqrt{s}}{2}(1,0,0,-1) .
\end{aligned}
$$

Then, the Mandelstam variables in the partonic level for the reaction $a+b \rightarrow c+d$ are expressed as

$\hat{s}=\left(p_{a}+p_{b}\right)^{2}=2 p_{a} \cdot p_{b}=x_{a} x_{b} s$,

$\hat{t}=\left(p_{a}-p_{c}\right)^{2}=Q^{2}-2 p_{a} \cdot q=Q^{2}+x_{a}\left(t-Q^{2}\right)$,

$\hat{u}=\left(p_{b}-p_{c}\right)^{2}=Q^{2}-2 p_{b} \cdot q=Q^{2}+x_{b}\left(u-Q^{2}\right)$.

Since the gluon transversity of the deuteron is studied in this work, we consider that the deuteron is linearly polarized with the polarization vectors $E_{x}$ and $E_{y}$ in Eq. (33). In probing the gluon transversity of the deuteron by the reaction $p+d \rightarrow \mu^{+} \mu^{-}+X$, this deuteron polarization needs to be considered with the unpolarized proton beam. In particular, the following combination of the polarized differential cross sections should be studied:

$$
d \sigma\left(E_{x}\right)-d \sigma\left(E_{y}\right) .
$$

Then, the linear-polarization tensor of Eqs. (38)-(40) appears in calculating the cross section asymmetry. Later, there appears the following contraction:

$$
q_{\mu} S_{\mathrm{TT}}^{\mu \nu} q_{\nu}=-q_{T}^{2} \cos (2 \phi)
$$


in calculating the cross section. Equation (54) is obtained by using the momentum assignments of Eq. (43).

\section{Parton correlation and distribution functions of proton and deuteron}

The cross section for $p+d \rightarrow \mu^{+} \mu^{-}+X$ is expressed in terms of parton correlation functions and subsequently by collinear PDFs, so that they are introduced in this section. Later, the cross section is expressed by the hadron tensor $W_{\mu \nu}$ multiplied by the photon tensor $L^{\mu \nu}$ in Sec. III D. The hadron tensor contains parton correlation functions in the proton and deuteron. There are correlation functions for quarks, antiquarks, and gluon $(h=q, \bar{q}, g)$ in the hadron $H$,

$\Phi_{h / H}=\Phi_{u / H}, \quad \Phi_{d / H}, \ldots, \quad \Phi_{\bar{u} / H}, \quad \Phi_{\bar{d} / H}, \ldots, \quad \Phi_{g / H}$.

The quark correlation functions are illustrated in Fig. 6, and they are defined by the matrix elements for quarks as

$$
\begin{aligned}
& \Phi_{q / H}^{i j}\left(p_{h}, p_{H}, s_{H}\right) \\
& \quad=\int \frac{d^{4} \xi_{h}}{(2 \pi)^{4}} e^{i p_{h} \cdot \xi_{h}}\left\langle p_{H} s_{H}\left|\bar{\psi}_{j}(0) \psi_{i}\left(\xi_{h}\right)\right| p_{H} s_{H}\right\rangle,
\end{aligned}
$$

where $\psi$ is the quark field, and $\xi_{h}$ is a four-dimensional space-time coordinate. To be precise, the gauge link should exist between $\bar{\psi}_{j}(0)$ and $\psi_{i}\left(\xi_{h}\right)$ to satisfy the color gauge invariance; however, they are not explicitly written in this paper. The correlation function indicates the amplitude to extract a parton from a hadron and then to insert it into the hadron at a different space-time point. The correlation function for the antiquark is given by changing $\bar{\psi}_{j}(0) \psi_{i}\left(\xi_{h}\right)$ for $\psi_{i}(0) \bar{\psi}_{j}\left(\xi_{h}\right)$,

$$
\begin{aligned}
& \Phi_{\bar{q} / H}^{i j}\left(p_{h}, p_{H}, s_{H}\right) \\
& \quad=\int \frac{d^{4} \xi_{h}}{(2 \pi)^{4}} e^{i p_{h} \cdot \xi_{h}}\left\langle p_{H} s_{H}\left|\psi_{i}(0) \bar{\psi}_{j}\left(\xi_{h}\right)\right| p_{H} s_{H}\right\rangle .
\end{aligned}
$$

The quark and antiquark correlation functions in Refs. [39,41,42] are the same as the one in Eqs. (56) and (57). However, they are defined without the $1 /(2 \pi)^{4}$ factor in Ref. [17].

The gluon correlation function is defined in the similar way by

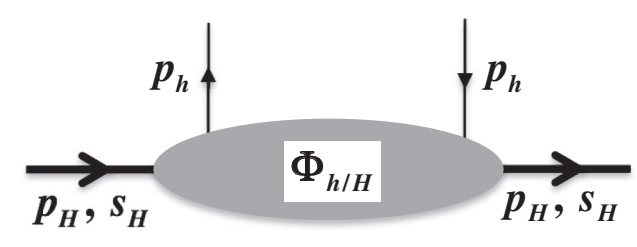

FIG. 6. Quark correlation function $\Phi_{h / H}$.

$$
\begin{aligned}
\Phi_{g / H}^{\alpha \beta}\left(p_{h}, p_{H}, s_{H}\right)= & N_{g / H} \int \frac{d^{4} \xi}{(2 \pi)^{4}} e^{i p_{h} \cdot \xi_{h}} \\
& \times\left\langle p_{H} s_{H}\left|A^{\alpha}(0) A^{\beta}(\xi)\right| p_{H} s_{H}\right\rangle,
\end{aligned}
$$

where $A^{\alpha}$ is given by $A^{\alpha}=A_{a}^{\alpha} t^{a}$ as explained below Eq. (22), and $N_{h / H}$ is the normalization constant. The gluon correlation function is often expressed by the gluonfield strength tensor $F_{a}^{\mu \nu}=\partial^{\mu} A_{a}^{\nu}-\partial^{\nu} A_{a}^{\mu}+g f_{a b c} A_{b}^{\mu} A_{c}^{\mu}$ $[17,41,42,45]$. Let us consider the hadron $H$ with momentum in the positive- $z$ direction. In the lightcone gauge $A^{+}=0$, the gluon filed has three components consist of $\vec{A}_{\perp}$ and $A^{-}$, and it satisfies $\partial^{+} A^{\mu}=F^{+\mu}$. Therefore, Eq. (58) becomes

$$
\begin{aligned}
\Phi_{g / H}^{\alpha \beta}\left(p_{h}, p_{H}, s_{H}\right)= & \frac{1}{N_{g / H}} \int \frac{d^{4} \xi}{(2 \pi)^{4}} e^{i p_{h} \cdot \xi_{h}} \\
& \times\left\langle p_{H} s_{H}\left|F^{+\alpha}(0) F^{+\beta}\left(\xi_{h}\right)\right| p_{H} s_{H}\right\rangle,
\end{aligned}
$$

where $F^{\mu \nu}$ is defined with the color factor as $F^{\mu \nu}=F_{a}^{\mu \nu} t^{a}$. One may note that the gluon correlation function in Eq. (59) is slightly different from the ones in Refs. [41,42] by the factor of $1 / N_{g / H}: \quad \Phi_{g / H}^{\alpha \beta}\left(p_{h}, p_{H}, s_{H}\right)_{\text {our }}=$ $\left(1 / N_{g / H}\right) \Phi_{g / H}^{\alpha \beta}\left(p_{h}, p_{H}, s_{H}\right)_{[41,42]}$. The overall normalization constant $N_{h / H}$ is different depending on the hadron momentum direction, namely in the positive- or negative- $z$ direction,

$$
N_{g / H}=\left\{\begin{array}{ll}
p_{h}^{+} & \text {for } H=A \\
p_{h}^{-} & \text {for } H=B
\end{array} .\right.
$$

Here, $p_{h}^{ \pm}$are the lightcone momenta. The details of this gluon normalization factor are explained in Ref. [46].

Next, we define TMDs and the collinear PDFs from the correlation functions. The correlation functions are integrated over $p_{h}\left(p_{a}\right.$ or $\left.p_{b}\right)$ to obtain the collinear correlation functions,

$$
\begin{aligned}
\Phi_{h / H}\left(x_{h}\right) & =\int d^{2} p_{h T} \Phi_{h / H}\left(x_{h}, p_{h T}\right) \\
& =\int d^{4} p_{h} \Phi_{h / H}\left(p_{h}, p_{H}, s_{H}\right) \delta\left(p_{h}^{ \pm}-x_{h} p_{H}^{ \pm}\right),
\end{aligned}
$$

where $\vec{p}_{h T}$ is the transverse momentum of the parton $h$, and $\Phi_{h / H}\left(x_{h}, \vec{p}_{h T}\right)$ are transverse-momentum-dependent correlation functions, which are related to the TMDs. The $+/-$ indicates the $\delta$ function $\delta\left(p_{h}^{+}-x_{h} p_{A}^{+}\right)$in the proton $A$ or $\delta\left(p_{h}^{-}-x_{h} p_{B}^{-}\right)$in the deuteron $B$. The notation \pm indicates $+(-)$ for $h=a$ and $H=A(h=b$ and $H=B)$.

There are some differences from other publication in kinematical factors $[17,39,41,42]$. The relations are 


$$
\begin{aligned}
& \Phi_{q / A}\left(p_{q}, p_{A}, s_{A}\right)=\Phi_{q / A}\left(p_{q}, p_{A}, s_{A}\right)_{[17,39,42]}, \\
& \Phi_{q / A}\left(x_{a}\right)=\Phi_{q / A}\left(x_{a}\right)_{[42]}=\frac{1}{p_{A}^{+}} \Phi_{q / A}\left(x_{a}\right)_{[17,39]},
\end{aligned}
$$

by taking $H=A$ for the quark correlation functions and the PDFs. The TMD relations are the same as the ones for the above PDFs. In the same way, there are differences for the gluon correlation function and its collinear distribution function as

$$
\begin{aligned}
& \Phi_{g / A}^{\alpha \beta}\left(p_{g}, p_{A}, s_{A}\right)=\frac{1}{p_{g / A}^{+}} \Phi_{g / A}^{\alpha \beta}\left(p_{g}, p_{A}, s_{A}\right)_{[39,41,42,47]}, \\
& \Phi_{g / A}^{\alpha \beta}\left(x_{a}\right)=\frac{1}{x_{a} p_{A}^{+}} \Phi_{g / A}^{\alpha \beta}\left(x_{a}\right)_{[42]}=\frac{1}{x_{a}} \Phi_{g / A}^{\alpha \beta}\left(x_{a}\right)_{[39,41,47]},
\end{aligned}
$$

where $p_{g / A}^{+}=x_{a} p_{A}^{+}$is used. Namely, the collinear correlation functions are defined as dimensionless quantities in this paper.

In this work, the collinear functions are considered for finding the gluon transversity, so that the parton momenta are integrated except for the + or - lightcone component as given in Eq. (61). Usually, all the possible distribution functions are listed in the TMD correlation-function form, so that the TMDs $f_{h / H}\left(x, \vec{p}_{h T}\right)$ are integrated over $\vec{p}_{h T}$ to become the PDFs $f_{h / H}(x)$ for our work,

$$
f_{h / H}(x)=\int d^{2} p_{h T} f_{h / H}\left(x, \vec{p}_{h T}\right)
$$

fThe quark and gluon correlation functions are expressed by the TMDs for the proton and deuteron [17,39,41,42], and they are integrated over $\vec{p}_{h T}$. Here, we are interested in probing the gluon transversity distribution of the deuteron. For finding it in the collinear formalism, we first consider the leading-twist part [17],

$$
\begin{aligned}
\Phi_{q / A}\left(x_{a}\right)= & \frac{1}{2}\left[\not h f_{1, q / A}\left(x_{a}\right)+\gamma_{5} \not h S_{A, L} g_{1, q / A}\left(x_{a}\right)\right. \\
& \left.+\not h \gamma_{5} \phi_{A, T} h_{1, q / A}\left(x_{a}\right)\right],
\end{aligned}
$$

as explained in Sec. III D. Here, $f_{1, q / A}\left(x_{a}\right), g_{1, q / A}\left(x_{a}\right)$, and $h_{1, q / A}\left(x_{a}\right)\left(\equiv \Delta_{T} q\left(x_{a}\right)\right.$ in this paper) are twist-2 distribution functions which indicate unpolarized, longitudinally polarized, and transversity distributions.

For the spin-1 deuteron, one should note that there are additional structure functions in comparison with the spin$1 / 2$ nucleon ones due to the spin- 1 nature. The twist-2 part of the quark correlation function for the spin-1 deuteron is given as $[39,42]$

$$
\begin{aligned}
\Phi_{q / B}\left(x_{b}\right)= & \frac{1}{2}\left[\not h f_{1, q / B}\left(x_{b}\right)+\gamma_{5} \not h S_{B, L} g_{1, q / B}\left(x_{b}\right)\right. \\
& +\not h \gamma_{5} \phi_{B, T} h_{1, q / B}\left(x_{b}\right)+\not h S_{B, L L} f_{1 L L, q / B}\left(x_{b}\right) \\
& \left.+\sigma_{\mu \nu} h^{\nu} S_{B, L T}^{\mu} h_{1 L T, q / B}\left(x_{b}\right)\right],
\end{aligned}
$$

where $f_{1, q / B}\left(x_{b}\right)$ is the unpolarized distribution function, $g_{1, q / B}\left(x_{b}\right)$ is the longitudinally polarized one, $h_{1, q / B}\left(x_{b}\right)$ $\left(=\Delta_{T} q\left(x_{b}\right)\right)$ is the transversity, and $f_{1 L L, q / B}\left(x_{b}\right)$ and $h_{1 L T, q / B}\left(x_{b}\right)$ are tensor-polarized ones of the deuteron. The last two terms with $S_{B, L L}$ and $S_{B, L T}$ exist due to the spin-1 nature of the deuteron, and they do not exist for the spin-1/2 proton. The correlation functions and the PDFs for antiquarks are obtained by the replacement $q \rightarrow \bar{q}$ in these expressions.

For the gluon correlation function in the deuteron, the twist-2 part is similarly given as $[41,42]$

$$
\begin{aligned}
\Phi_{g / B}^{\alpha \beta}\left(x_{b}\right) \equiv & \int d^{2} p_{b T} \Phi_{g / B}^{\alpha \beta}\left(x, \vec{p}_{b T}\right) \\
= & \frac{1}{2}\left[-g_{T}^{\alpha \beta} f_{1, g / B}\left(x_{b}\right)+i \epsilon_{T}^{\alpha \beta} S_{B, L} g_{1, g / B}\left(x_{b}\right)\right. \\
& -g_{T}^{\alpha \beta} S_{B, L L} f_{1 L L, g / B}\left(x_{b}\right) \\
& \left.+S_{B, \mathrm{TT}}^{\alpha \beta} h_{1 \mathrm{TT}, g / B}\left(x_{b}\right)\right],
\end{aligned}
$$

where $f_{1, g / B}$ is the unpolarized gluon distribution function, $g_{1, g / B}$ is the longitudinally polarized one, $f_{1 L L, g / B}$ and $h_{1 \mathrm{TT}, g / B}[41,42]\left(\equiv-\Delta_{T} g_{B}\right.$ in this paper) are tensor- and linearly polarized ones. We have a negative sign for $\Delta_{T} g_{B}$ because the linear polarization for the gluon transversity is defined by $\varepsilon_{x}-\varepsilon_{y}$ in Eq. (39) instead of $\varepsilon_{y}-\varepsilon_{x}$ in Refs. [41,42]. There are different definitions on the gluon transversity and its notation,

$$
\begin{aligned}
\Delta_{2} G(x) & =g_{\hat{x} / \hat{x}}(x)-g_{\hat{y} / \hat{x}}(x) \quad[13,47], \\
a(x) & =g_{\hat{x} / \hat{x}}(x)-g_{\hat{y} / \hat{x}}(x) \quad[25,27], \\
\Delta_{L} g(x) & =g_{\hat{x} / \hat{x}}(x)-g_{\hat{y} / \hat{x}}(x) \quad[20], \\
\delta G(x) & =-g_{\hat{x} / \hat{x}}(x)+g_{\hat{y} / \hat{x}}(x) \quad[28,48], \\
h_{1 \mathrm{TT}, g}(x) & =-g_{\hat{x} / \hat{x}}(x)+g_{\hat{y} / \hat{x}}(x) \quad[39,41,49], \\
\Delta_{T} g(x) & =g_{\hat{x} / \hat{x}}(x)-g_{\hat{y} / \hat{x}}(x) \quad[50], \quad \text { this work, }
\end{aligned}
$$

where $g_{\hat{h} / \hat{H}}$ indicates the gluon distribution with the gluon polarization $\hat{h}$ and the hadron (deuteron) polarization $\hat{H}$. In Eq. (67), the notation $\epsilon_{T}^{\alpha \beta}$ is defined by $\epsilon_{T}^{\alpha \beta} \equiv \epsilon^{\alpha \beta-+}$ $\left(\epsilon_{T}^{12}=-\epsilon_{T}^{21}=1\right.$, others $\left.=0\right)$. It is different from the linear-polarization tensor $\varepsilon_{\mathrm{TT}}^{\alpha \beta}$. The gluon correlation function $\Phi_{g / B}^{\alpha \beta}(x)$ is expressed by only the transverse indices $\alpha$ and $\beta(=1,2)$, because other components do not contribute to the cross section in the leading-twist level. The gluon correlation function $\Phi_{g / A}^{\alpha \beta}(x)$ of the proton is the same as the 
one for the deuteron in Eq. (67) by terminating the tensor terms $\left(S_{L L}=S_{\mathrm{TT}}^{\alpha \beta}=0\right)$. However, its expression is not explicitly written because it does not contribute to the Drell-Yan cross section in our current linear-polarization asymmetry as the leading one.

\section{Cross section for Drell-Yan process $p+d \rightarrow \mu^{+} \mu^{-}+X$ in parton model}

The cross section for the proton-deuteron Drell-Yan process $p+d \rightarrow \mu^{+} \mu^{-}+X$ of Fig. 5(a) is calculated by the partonic-subprocess cross section $\hat{\sigma}_{a b \rightarrow c d}$ in Fig. 5(b). The partonic cross section is convoluted with the PDFs of hadrons, as explained in Ref. [43] for the unpolarized cross section. Especially, since the correlation-function formalism is used in deriving the polarized cross section, we first show the Drell-Yan cross section in terms of the parton correlation functions.

We investigate the dimuon production with finite $q_{T}$. First, the $q($ in $p) \bar{q}($ in $d)$ subprocess contribution is considered as an example to confirm our formalism with the correlation functions. The unpolarized $p d$ Drell-Yan cross section $d \sigma_{p d \rightarrow \gamma X}$ is expressed by the partonic cross section for $q(p) \bar{q}(d) \rightarrow \gamma^{*} g \rightarrow \mu^{+} \mu^{-} g$ by the convolution with the quark and antiquark distribution functions as

$$
\begin{aligned}
\left.d \sigma_{p d \rightarrow \mu^{+} \mu^{-} X}\right|_{q \bar{q} \rightarrow \gamma^{*} g}= & \int_{0}^{1} d x_{a} \int_{0}^{1} d x_{b} q\left(x_{a}\right) \bar{q}\left(x_{b}\right) \\
& \times d \hat{\sigma}_{q \bar{q} \rightarrow \mu^{+} \mu^{-} g} .
\end{aligned}
$$

The partonic cross section for $q \bar{q} \rightarrow \gamma^{*} g \rightarrow \mu^{+} \mu^{-} g$ is given by

$$
\begin{aligned}
d \hat{\sigma}_{q \bar{q} \rightarrow \mu^{+} \mu^{-} g}= & \frac{1}{4 p_{a} \cdot p_{b}} \sum_{\substack{\text { spini, } \\
\text { color }}} \sum_{\text {lavor }}\left|M_{q \bar{q} \rightarrow \gamma^{*} g \rightarrow \mu^{+} \mu^{-} g}\right|^{2} \\
& \times(2 \pi)^{4} \delta^{4}\left(p_{a}+p_{b}-k_{1}-k_{2}-p_{d}\right) \\
& \times \frac{d^{3} k_{1}}{2 E_{1}(2 \pi)^{3}} \frac{d^{3} k_{2}}{2 E_{2}(2 \pi)^{3}} \frac{d^{3} p_{d}}{2 E_{d}(2 \pi)^{3}},
\end{aligned}
$$

where $k_{1}, k_{2}$, and $p_{d}$ are momenta for $\mu^{-}, \mu^{+}$, and gluon in the final state. The three-body phase space for the final state is written by the two-body phase spaces as [51]

$$
\begin{aligned}
& d \Phi_{3}\left(p_{a}+p_{b} ; k_{1}, k_{2}, p_{d}\right) \\
& \quad=d \Phi_{2}\left(q ; k_{1}, k_{2}\right) d \Phi_{2}\left(p_{a}+p_{b} ; q, p_{d}\right),
\end{aligned}
$$

where the $n$-body phase space is defined by

$$
d \Phi_{n}\left(P ; p_{1}, \ldots, p_{n}\right)=\delta^{4}\left(P-\sum_{i=1}^{n} p_{i}\right) \prod_{i=1}^{n} \frac{d^{3} p_{i}}{2 E_{i}(2 \pi)^{3}} .
$$

The matrix element is described by the process $q \bar{q} \rightarrow \gamma^{*} g$ and subsequent $\gamma^{*} \rightarrow \mu^{+} \mu^{-}$as

$$
\begin{aligned}
M_{q \bar{q} \rightarrow \gamma^{*} g \rightarrow \mu^{+} \mu^{-} g} & =e M_{\gamma^{*} \rightarrow \mu^{+} \mu^{-}}^{\mu} \frac{-1}{Q^{2}} e M_{q \bar{q} \rightarrow \gamma^{*} g, \mu}, \\
M_{\gamma^{*} \rightarrow \mu^{+} \mu^{-}}^{\mu} & =\bar{u}\left(k_{1}, \lambda_{1}\right) \gamma^{\mu} v\left(k_{2}, \lambda_{2}\right), \\
M_{q \bar{q} \rightarrow \gamma^{*} g, \mu} & =e_{q} \varepsilon^{* \alpha}\left(p_{d}, \lambda_{d}\right) \bar{v}\left(p_{b}, \lambda_{b}\right) \Gamma_{\mu \alpha} u\left(p_{a}, \lambda_{a}\right),
\end{aligned}
$$

where $\Gamma_{\mu \alpha}$ indicates the $q \bar{q} \gamma g$ interaction part. The dimuon term is calculated and it becomes the lepton tensor $L^{\mu \nu}$,

$$
\begin{aligned}
& \sum_{\lambda_{1}, \lambda_{2}}\left(M_{\gamma^{*} \rightarrow \mu^{+} \mu^{-}}^{\mu}\right)^{\dagger} M_{\gamma^{*} \rightarrow \mu^{+} \mu^{-}}^{\nu} \\
& \quad=2 L^{\mu \nu}=4\left(k_{1}^{\mu} k_{2}^{\nu}+k_{1}^{\nu} k_{2}^{\mu}-k_{1} \cdot k_{2} g^{\mu \nu}\right),
\end{aligned}
$$

where the overall factor of 2 is assigned so that the lepton tensor agrees with the conventional one used in describing the deep inelastic lepton-nucleon scattering. Its integral over the phase space is given by

$$
\int d \Phi_{2}\left(q ; k_{1}, k_{2}\right) 2 L^{\mu \nu}=\frac{1}{6 \pi}\left(q^{\mu} q^{\nu}-Q^{2} g^{\mu \nu}\right) .
$$

This relation eventually becomes $-Q^{2} g^{\mu \nu} /(6 \pi)$ by considering the current conservation $q^{\mu} M_{q \bar{q} \rightarrow \gamma^{*} g, \mu}=0$.

From the relations in Eq. (52), the four-momentum square $p_{d}^{2}$ becomes

$p_{d}^{2}=\left(p_{a}+p_{b}-q\right)^{2}=s\left(x_{a}-x_{1}\right)\left[x_{b}-\frac{x_{a} x_{2}-\tau}{x_{a}-x_{1}}\right]$,

where the used kinematical variables $\left(x_{1}, x_{2}, \ldots\right)$ are defined in Sec. III B. Then, using the relations for the integrals

$$
\begin{gathered}
\frac{d^{3} q}{2 E}=\frac{1}{4} d q_{T}^{2} d \phi d y, \quad \frac{d^{3} p_{d}}{2 E_{d}}=d^{4} p_{d} \delta\left(p_{d}^{2}\right), \\
\int_{0}^{1} d x_{b} \delta\left(p_{d}^{2}\right)=\frac{1}{s\left(x_{a}-x_{1}\right)}
\end{gathered}
$$

and taking the gluon-spin summation, we finally obtain the cross section expression as

$$
\begin{aligned}
& \left.\frac{d \sigma_{p d \rightarrow \mu^{+} \mu^{-} X}}{d \tau d q_{T}^{2} d \phi d y}\right|_{q \bar{q} \rightarrow \gamma^{*} g}=\frac{\alpha^{2}}{12(2 \pi)^{2} Q^{2}} \sum_{\substack{\text { spin, } \\
\text { color }}} \sum_{q} e_{q}^{2} g^{\mu \nu} g^{\alpha \beta} \\
& \times \int_{\min \left(x_{a}\right)}^{1} \frac{1}{x_{a}-x_{1}} q_{A}\left(x_{a}\right) \bar{q}_{B}\left(x_{b}\right) \operatorname{Tr}\left[\frac{\gamma^{+}}{2} \Gamma_{\nu \beta} \frac{\gamma^{-}}{2} \hat{\Gamma}_{\mu \alpha}\right] .
\end{aligned}
$$

Here, due to the $\delta$ function for $p_{d}^{2}$ in Eqs. (76) and (77) and also the kinematical constraint of $\max \left(x_{b}\right)=1$, we have the relations

$$
x_{b}=\frac{x_{a} x_{2}-\tau}{x_{a}-\tau}, \quad \min \left(x_{a}\right)=\frac{x_{1}-\tau}{1-x_{2}},
$$

and the notation $\hat{O}$ is defined by 


$$
\hat{O} \equiv \gamma^{0} O^{\dagger} \gamma^{0}
$$

The fine structure constant $\alpha$ is given by $\alpha=e^{2} /(4 \pi)$.

Next, we try to write the cross section in terms of parton correlation functions in Sec. III C by considering only the subprocess $q(p)+\bar{q}(d) \rightarrow \gamma+g$ in Fig. 7 as

$$
\begin{aligned}
\left.d \sigma_{p d \rightarrow \gamma X}\right|_{q \bar{q} \rightarrow \gamma^{*} g}= & \frac{1}{4 p_{A} \cdot p_{B}} \int \frac{d^{4} p_{a}}{(2 \pi)^{4}} \int \frac{d^{4} p_{b}}{(2 \pi)^{4}} \sum_{\substack{\text { spin, } \\
\text { color }}} \sum_{\text {flavor }} \sum_{X_{A}, X_{B}}(2 \pi)^{4} \delta^{4}\left(p_{A}-p_{a}-p_{A X}\right)(2 \pi)^{4} \delta^{4}\left(p_{B}-p_{b}-p_{B X}\right) \\
& \times\left|\left\langle X_{B}\left|\bar{\psi}_{b, l}(0)\right| p_{B} s_{B}\right\rangle\left(\Gamma_{q \bar{q} \rightarrow \gamma^{*} g, \mu}\right)_{l k}\left\langle X_{A}\left|\psi_{a, k}(0)\right| p_{A} s_{A}\right\rangle M_{\gamma^{*} \rightarrow \mu^{+} \mu^{-}}\right|^{2} \\
& \times\left(\frac{-e}{Q^{2}}\right)^{2}(2 \pi)^{4} \delta^{4}\left(p_{a}+p_{b}-k_{1}-k_{2}-p_{d}\right) \frac{d^{3} k_{1}}{2 E_{1}(2 \pi)^{3}} \frac{d^{3} k_{2}}{2 E_{2}(2 \pi)^{3}} \frac{d^{3} p_{d}}{2 E_{d}(2 \pi)^{3}},
\end{aligned}
$$

where the spin summations are taken for muons, quark, antiquark, and gluon. The parton-interaction part $\Gamma_{q \bar{q} \rightarrow \gamma^{*} g, \mu}$ is given by $\Gamma_{q \bar{q} \rightarrow \gamma^{*} g, \mu}=e_{q} \varepsilon^{* \alpha}\left(p_{d}, \lambda_{d}\right) \Gamma_{\mu \alpha}$ by extracting out the quark charge $e_{q}$ and the gluonpolarization vector $\varepsilon^{* \alpha}\left(p_{d}, \lambda_{d}\right)$ from $\Gamma_{q \bar{q} \rightarrow \gamma^{*} g, \mu}$. By changing the three-body phase space to the two-body ones and repeating the same calculations from Eq. (74) to Eq. (77), the cross section is written by the lepton tensor $L^{\mu \nu}$ in Eqs. (74) and (75) multiplied by the hadron tensor $W_{\mu \nu}$,

$$
\begin{aligned}
& \frac{d \sigma_{p d \rightarrow \mu^{+} \mu^{-} X}}{d \tau d q_{T}^{2} d \phi d y} \\
& \quad=\frac{\alpha^{2}}{2(2 \pi)^{2} Q^{4}}\left(\int d \Phi_{2}\left(q ; k_{1}, k_{2}\right) 2 L^{\mu \nu}\right) W_{\mu \nu} .
\end{aligned}
$$

This cross section expression, in terms of lepton and hadron tensors, can be used not only for the process $q \bar{q} \rightarrow \gamma^{*} g$ under consideration but also for any partonic subprocesses in the Drell-Yan process. The hadron tensor is given by

$$
\begin{aligned}
W_{\mu \nu}(q \bar{q})= & \int \frac{d^{4} p_{a}}{(2 \pi)^{4}} \int \frac{d^{4} p_{b}}{(2 \pi)^{4}} \sum_{\substack{\text { spin, } \\
\text { color }}} \sum_{q} \sum_{X_{A}, X_{B}} e_{q}^{2}(2 \pi)^{4} \delta^{4}\left(p_{A}-p_{a}-p_{A X}\right)(2 \pi)^{4} \delta^{4}\left(p_{B}-p_{b}-p_{B X}\right) \\
& \times\left[\left\langle X_{B}\left|\bar{\psi}_{b, j}(0)\right| p_{B} s_{B}\right\rangle\left(\Gamma_{q \bar{q} \rightarrow \gamma^{*} g, \mu}\right)_{j i}\left\langle X_{A}\left|\psi_{a, i}(0)\right| p_{A} s_{A}\right\rangle\right]^{\dagger}\left[\left\langle X_{B}\left|\bar{\psi}_{b, l}(0)\right| p_{B} s_{B}\right\rangle\left(\Gamma_{q \bar{q} \rightarrow \gamma^{*} g, \nu}\right)_{l k}\left\langle X_{A}\left|\psi_{a, k}(0)\right| p_{A} s_{A}\right\rangle\right] \\
& \times(2 \pi)^{4} \delta^{4}\left(p_{a}+p_{b}-q-p_{d}\right) \frac{d^{3} p_{d}}{2 E_{d}(2 \pi)^{3}},
\end{aligned}
$$

for the $q \bar{q} \rightarrow \gamma^{*} g$ process.

We try to write the hadron tensor in terms of the correlation functions. The $\delta$ functions $\delta^{4}\left(p_{H}-p_{h}-p_{H_{X}}\right)$ ( $H=A$ or $B, h=a$ or $b$ ) in Eq. (83) are expressed by the

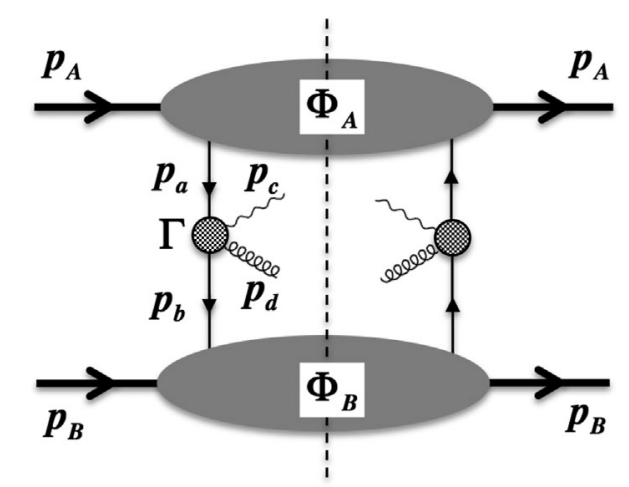

FIG. 7. $q+\bar{q} \rightarrow \gamma^{*}+g$ process for cross section of $p+d \rightarrow \gamma^{*}+X$. integrals of exponential functions $(2 \pi)^{4} \delta^{4}\left(p_{H}-p_{h}-\right.$ $\left.p_{H_{X}}\right)=\int d^{4} \xi_{h} e^{-i\left(p_{H}-p_{h}-p_{H_{X}}\right) \cdot \xi_{h}}$. Then, the quark field is given at $\xi_{h}$ in the matrix elements with the exponential factor as $e^{i p_{H_{X}} \cdot \xi_{h}} \psi(0) e^{-i p_{H} \cdot \xi_{h}}=\psi\left(\xi_{h}\right)$. The completeness relation $\sum_{X_{H}}\left|X_{H}\right\rangle\left\langle X_{H}\right|=1$ is used to express the hadron tensor in terms of parton correlation functions $\Phi_{h / H}\left(p_{h}, p_{H}, s_{H}\right)$, which include parton-spin summations and averages, as

$$
\begin{aligned}
W_{\mu \nu}(q \bar{q})= & 2 \pi \sum_{\lambda_{d}} \overline{\sum_{\text {color }}} \sum_{q} e_{q}^{2} \int d^{4} p_{a} \int d^{4} p_{b} \delta\left(p_{d}^{2}\right) \\
& \times \operatorname{Tr}\left[\Gamma_{q \bar{q} \rightarrow \gamma^{*} g, \nu} \Phi_{q / A}\left(p_{a}, p_{A}, s_{A}\right)\right. \\
& \left.\times \hat{\Gamma}_{q \bar{q} \rightarrow \gamma^{*} g, \mu} \Phi_{\bar{q} / B}\left(p_{b}, p_{B}, s_{B}\right)\right] .
\end{aligned}
$$

Here, the summation is taken over the gluon spin $\lambda_{d}$. In this way, the cross section for $p+d \rightarrow \gamma^{*}+X$ is generally expressed by the parton correlation functions defined by the 
matrix elements of the bilocal operators in Eqs. (56) and (57).

Using the integrated collinear correlation functions with the lightcone relations $d^{4} p_{h}=d p_{h}^{+} d p_{h}^{-} d^{2} p_{h T}$ and $p_{h}^{ \pm}=x_{h} p_{H}^{ \pm}$, and using the integral $\int d x_{b} \delta\left(p_{d}^{2}\right)$ of Eq. (76), we have the hadron tensor,

$$
\begin{aligned}
W_{\mu \nu}(q \bar{q})= & \sum_{\lambda_{d}} \sum_{\text {color }} \sum_{q} e_{q}^{2} \int_{\min \left(x_{a}\right)}^{1} d x_{a} \frac{\pi}{x_{a}-x_{1}} \\
& \times \operatorname{Tr}\left[\Gamma_{q \bar{q} \rightarrow \gamma^{*} g, \nu} \Phi_{q / A}\left(x_{a}\right) \hat{\Gamma}_{q \bar{q} \rightarrow \gamma^{*} g, \mu} \Phi_{\bar{q} / B}\left(x_{b}\right)\right] .
\end{aligned}
$$

The momentum fraction $x_{b}$ for a parton in the deuteron is defined in Eq. (51), so its upper bound is one. However, one should be careful about this kinematical region. In lepton DIS, the Bjorken scaling variable for the nucleon $x_{B j}=$ $Q^{2} /\left(2 M_{N} \nu\right)$ is usually used also for the deuteron. In this case, due to the difference between the nucleon and deuteron masses $\left(M_{d} / M_{N} \simeq 2\right)$, the upper bound is $\max \left(x_{B j}\right) \simeq 2$. One should note this convention difference in numerical estimates. If we provide the correlation functions $\Phi_{q / A}\left(x_{a}\right), \Phi_{\bar{q} / B}\left(x_{b}\right)$ and the parton-interaction part $\Gamma_{q \bar{q} \rightarrow \gamma^{*} g, \mu}$ in Eq. (85), the Drell-Yan cross section can be evaluated. We explained relevant PDFs of the proton and deuteron in connection with their parton correlation functions in Sec. III C for evaluating the partonic cross section in Sec. III E. From the hadron tensor of Eq. (85) with the unpolarized correlation functions $\Phi_{q / A}\left(x_{a}\right)=\bar{x} f_{q / A}\left(x_{a}\right) / 2$ and $\Phi_{\bar{q} / A}\left(x_{b}\right)=\not h f_{\bar{q} / B}\left(x_{b}\right) / 2$ with $\bar{x}=\gamma^{-}$and $\not h=\gamma^{+}$, the cross section of Eq. (82) becomes identical to Eq. (78).

Before stepping into the cross-section calculation with the gluon transversity, we comment on the factorization of the cross section into the soft-physics part of the correlation functions and the hard part described by perturbative QCD. As it is explained in Ref. [52], the Drell-Yan cross sections are factorized in the leading order (LO) of $\alpha_{s}$ if the hard scale $Q^{2}$ is reasonably large. The factorization is satisfied in the reasonably large $Q^{2}$ region, so that it is theoretically meaningful to extract the gluon transversity distribution from experimental measurements.

In this work, the linear-polarization asymmetry $E_{x}-E_{y}$, is taken for the deuteron as illustrated in Fig. 8, so that only the $h_{1 \mathrm{TT}, g / B}(x)$ ( $\equiv-\Delta_{T} g_{B}(x)$ in this work) term of Eq. (67)

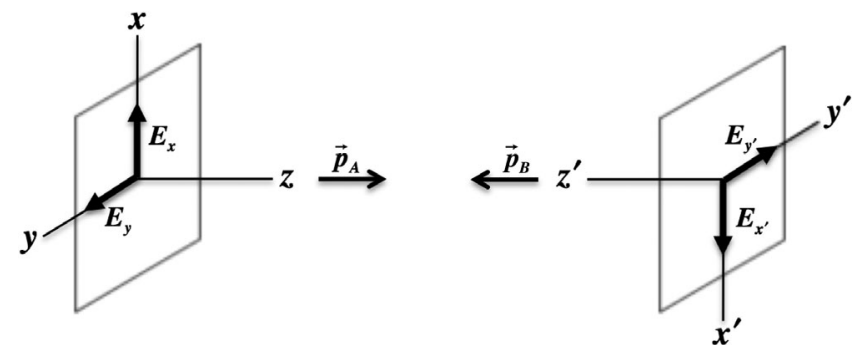

FIG. 8. Linear polarizations $E_{x^{\prime}}$ and $E_{y^{\prime}}$ of spin-1 deuteron in proton $(A)$-deuteron $(B)$ collision. contributes. Actually, the asymmetry is $E_{x^{\prime}}-E_{y^{\prime}}$ because the deuteron moves in the negative- $z$ direction in the c.m. frame as shown in Fig. 8. However, according to Eq. (39), by changing the gluon polarization $\varepsilon$ for the deuteron one $E$, they are same: $E_{x^{\prime}}-E_{y^{\prime}}=E_{-x}-E_{-y}=E_{x}-E_{y}$. Namely, we leave only the $S_{B, \mathrm{TT}}^{\alpha \beta}$ term and terminate other unpolarized and spin-dependent ones, as explained in the end of Sec. III A, in the correlation function of Eq. (67). In the collinear formalism, the hadron tensor is given in the same way with Eq. (85) by

$$
\begin{aligned}
& W_{\mu \nu}\left(E_{x}-E_{y}\right) \\
& =\sum_{\lambda_{d}} \sum_{\text {color }} \sum_{q} e_{q}^{2} \int_{\min \left(x_{a}\right)}^{1} d x_{a} \frac{\pi}{p_{g}^{-}\left(x_{a}-x_{1}\right)} \\
& \quad \times \operatorname{Tr}\left[\Gamma_{\nu \beta}\left\{\Phi_{q / A}\left(x_{a}\right)+\Phi_{\bar{q} / A}\left(x_{a}\right)\right\} \hat{\Gamma}_{\mu \alpha} \Phi_{g / B}^{\alpha \beta}\left(x_{b}\right)\right],
\end{aligned}
$$

as illustrated in Fig. 9. The summation is taken over the quark spin $\lambda_{d}$. Here, only the linear polarization is considered for the deuteron by taking the $S_{B, \mathrm{TT}}^{\alpha \beta}$ term as the only one to a finite spin asymmetry. Then, the only contribution comes from the gluon transversity $\Delta_{T} g_{B}(x)$ in the deuteron. There is no such polarization term in the quark and antiquark distributions of the deuteron according to Eq. (66). In order to have the virtual photon in the intermediate stage, a charged parton, namely a quark or an antiquark, needs to be involved in the reaction, so that only quark and antiquark correlation functions contribute as the leading process from the proton. The collinear correlation functions in Eqs. (65) and (67) are used for calculating the hadron tensor of Eq. (86). Then, we find that the only contribution from the proton part should be the twist-2 PDF term $f_{1, q / A}$.

In this case of polarization, the trace of Eq. (86) typically looks like

$\operatorname{Tr}\left[\not \not_{d} \gamma_{\beta} \not \not_{i} \gamma_{\nu}\left\{\Phi_{q / A}\left(x_{a}\right)+\Phi_{\bar{q} / A}\left(x_{a}\right)\right\} \cdots \times \gamma_{\mu} \not \not_{i} \gamma_{\alpha} \Phi_{g / B}^{\alpha \beta}\left(x_{b}\right)\right]$,

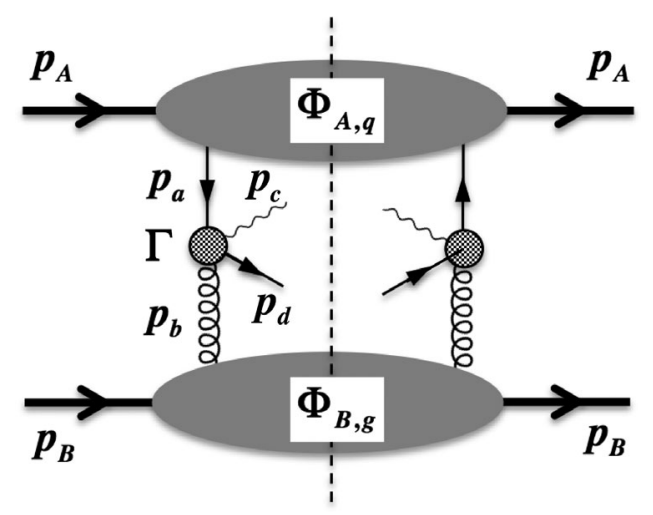

FIG. 9. Quark-gluon process contribution to the cross section. 
where $\gamma_{\alpha}$ and $\gamma_{\beta}$ are from the quark-gluon vertex, and $p_{i}$ is the intermediate-quark momentum, for example, $p_{i}=p_{a}+p_{b}$. There could be a factor $\not p_{a}$, for example, in an unpolarized-proton reaction. However, it is included into the definition of $\Phi_{q / A}$, and it corresponds to the first $\bar{x}$ in Eq. (65). There are seven $\gamma$ matrices, except for the ones in $\Phi_{q(\bar{q}) / A}$, in the trace and there is no $\gamma$ factor in the gluon transversity term $\Delta_{T} g_{B}\left(x_{b}\right)$ of $\Phi_{g / B}$, so that only odd numbers of $\gamma$ in $\Phi_{q / A}(x)$ contribute to the spin asymmetry. In addition, the unpolarized-proton beam is assumed in this work, so that only the unpolarized distribution $\bar{n} f_{1, q / A}\left(x_{a}\right) / 2$ contributes to the hadron tensor.

In this way, we find that the leading contribution starts from the twist-2 distribution $f_{1, q / A}$ in the nucleon part by considering that only the gluon transversity $\Delta_{T} g_{B}$ participates from the deuteron for the linear-polarization asymmetry. From Eqs. (65) and (67), they are given by

$$
\begin{aligned}
& \Phi_{q / A}\left(x_{a}\right)=\frac{1}{2} \not h q_{A}\left(x_{a}\right), \\
& \Phi_{g / B}^{\alpha \beta}\left(x_{b}\right)=-S_{B, \mathrm{TT}}^{\alpha \beta} \Delta_{T} g_{B}\left(x_{b}\right) .
\end{aligned}
$$

Here, $\Phi_{g / B}^{\alpha \beta}\left(x_{b}\right)$ is multiplied by the factor of 2 because the linear polarization $E_{x}-E_{y}$ is taken for showing the cross section instead of $\left(E_{x}-E_{y}\right) / 2$, and $f_{1, q / A}\left(x_{a}\right)$ is denoted as $q_{A}\left(x_{a}\right)$.

\section{E. Parton-interaction processes}

For calculating the hadron tensor of Eq. (86) and subsequently the cross section of Eq. (82), partonic matrix elements are calculated in this section. We consider the leading partonic processes in Fig. 10. Since the linearpolarization spin asymmetry in Eq. (53) is studied in this work and since unpolarized and polarized gluon distributions in the proton do not contribute as the leading process, main contributions come from the partonic reactions of

$$
q / \bar{q} \text { in proton }+g \text { in deuteron } \rightarrow \gamma^{*}+q,
$$

as shown in Fig. 10.

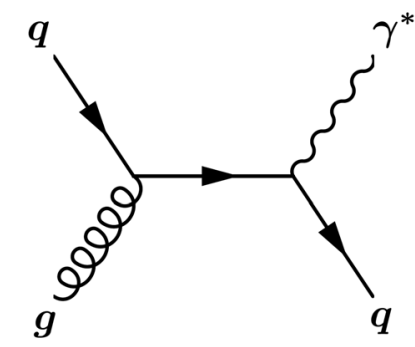

(1)

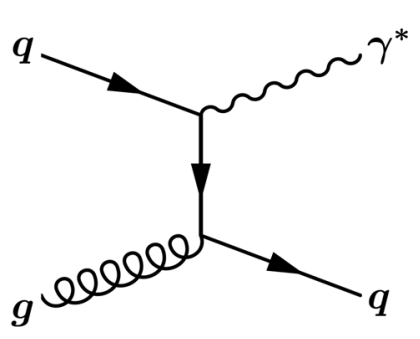

(2)
FIG. 10. Leading partonic processes $q\left(p_{a}\right)+g\left(p_{b}\right) \rightarrow$ $\gamma^{*}\left(p_{c}=q\right)+q\left(p_{d}\right)$ : (1) $s$-channel and (2) $t$-channel processes.
The parton-scattering amplitude of Fig. 10 is given by

$$
\begin{aligned}
\Gamma_{\mu \alpha} & =i g\left(t^{a}\right)_{j i} \bar{u}\left(p_{d}\right) G_{\mu \alpha}, \\
G_{\mu \alpha} & \equiv \gamma_{\mu} \frac{\not \phi_{a}+\not p_{b}}{\hat{s}} \gamma_{\alpha}+\gamma_{\alpha} \frac{\not p_{a}-\not q}{\hat{t}} \gamma_{\mu},
\end{aligned}
$$

where $g$ is the strong coupling constant. Then, the color factor in $W_{\mu \nu}$ becomes

$$
\begin{aligned}
\sum_{\text {color }}\left(t^{a}\right)_{j i}\left(t^{a}\right)_{j i}^{\dagger} & =\frac{1}{3 \cdot 8} \sum_{a}\left(t^{a}\right)_{j i}\left(t^{a}\right)_{j i}^{\dagger}=\frac{1}{8} C_{F}, \\
C_{F} & =\frac{N_{c}^{2}-1}{2 N_{c}}, \quad N_{c}=3 .
\end{aligned}
$$

Using these expressions, the running coupling constant given by $\alpha_{s}=g^{2} /(4 \pi), p_{g}^{-}=x_{b} p_{B}^{-}$, and the correlation functions in Eq. (88), we write the hadron tensor of Eq. (86) in terms of the PDFs as

$$
\begin{aligned}
W_{\mu \nu}\left(E_{x}-E_{y}\right)= & -\frac{\pi^{2} \alpha_{s} C_{F}}{4 p_{B}^{-}} \sum_{q} e_{q}^{2} \\
& \times \int_{\min \left(x_{a}\right)}^{1} d x_{a} \frac{1}{x_{b}\left(x_{a}-x_{1}\right)}\left\{q_{A}\left(x_{a}\right)+\bar{q}_{A}\left(x_{a}\right)\right\} \\
& \times \Delta_{T} g_{B}\left(x_{b}\right) S_{B, \operatorname{TT}}^{\alpha \beta} \operatorname{Tr}\left[\not p_{d} G_{\nu \beta} \not h \hat{G}_{\mu \alpha}\right] .
\end{aligned}
$$

Next, the trace should be calculated. We need trace calculations with eight $\gamma$ matrices and it is slightly lengthy, so that their results should be carefully checked. Three independent methods are used. First, we analytically calculated the trace by noting the $\mu \nu$ and $\alpha \beta$ symmetries of $g^{\mu \nu}$ and $S_{B, \mathrm{TT}}^{\alpha \beta}$ together with properties of spin and momentum vectors within the trace. As the independent second method, it is calculated by using the FeynCalc code [53] together with Mathematica. The third one is by using the Tracer code [54] with also Mathematica. All these results are consistent with each other.

We divide the trace into $s$-channel, $t$-channel, and their interference terms,

$$
\begin{aligned}
g^{\mu \nu} & S_{B, \mathrm{TT}}^{\alpha \beta} \operatorname{Tr}\left[\not \not_{d} G_{\nu \beta} \not \vec{h} \hat{G}_{\mu \alpha}\right] \\
& =\frac{1}{\hat{s}^{2}}\left|M_{s}\right|^{2}+\frac{1}{\hat{t}^{2}}\left|M_{t}\right|^{2}+\frac{1}{\hat{s} \hat{t}}\left(M_{s}^{*} M_{t}+M_{s} M_{t}^{*}\right) .
\end{aligned}
$$

Each term is calculated as

$$
\begin{aligned}
\left|M_{s}\right|^{2} & =g^{\mu \nu} S_{B, \mathrm{TT}}^{\alpha \beta} \operatorname{Tr}\left[\not \bar{n} \gamma_{\alpha}\left(\not p_{a}+\not p_{b}\right) \gamma_{\mu} \not p_{d} \gamma_{\nu}\left(\not \not a_{a}+\not p_{b}\right) \gamma_{\beta}\right] \\
& =16 \hat{s} \bar{n}^{\zeta} S_{B, \mathrm{TT}, \zeta}^{\alpha} p_{d, \alpha}=0, \\
\left|M_{t}\right|^{2} & =g^{\mu \nu} S_{B, \mathrm{TT}}^{\alpha \beta} \operatorname{Tr}\left[\bar{n} \gamma_{\mu}\left(\not p_{a}-\not \not_{c}\right) \gamma_{\alpha} \not \not_{d} \gamma_{\beta}\left(\not p_{a}-\not p_{c}\right) \gamma_{\nu}\right] \\
& =-32\left(q_{\alpha} S_{B, \mathrm{TT}}^{\alpha \beta} q_{\beta}\right) \bar{n} \cdot\left(p_{a}-p_{c}\right),
\end{aligned}
$$




$$
\begin{aligned}
& M_{s}^{*} M_{t}+M_{s} M_{t}^{*} \\
& =g^{\mu \nu} S_{B, \operatorname{TT}}^{\alpha \beta}\left\{\operatorname{Tr}\left[\not \vec{h} \gamma_{\alpha}\left(\not p_{a}+\not \not_{b}\right) \gamma_{\mu} \not \not_{d} \gamma_{\beta}\left(\not \not_{a}-\not \not_{c}\right) \gamma_{\nu}\right]\right. \\
& \left.+\operatorname{Tr}\left[\not h \gamma_{\mu}\left(\not \not_{a}-\not p_{c}\right) \gamma_{\alpha} \not p_{d} \gamma_{\nu}\left(\not p_{a}+\not \not_{b}\right) \gamma_{\beta}\right]\right\} \\
& =32\left(q_{\alpha} S_{B, \mathrm{TT}}^{\alpha \beta} q_{\beta}\right) \bar{n} \cdot\left(p_{a}+p_{b}\right) \text {. }
\end{aligned}
$$

Noting Eq. (54) and the relations $\bar{n} \cdot\left(p_{a}+p_{b}\right)=\sqrt{s / 2} x_{b}$ and $\bar{n} \cdot\left(p_{a}-p_{c}\right)=-\sqrt{s / 2} x_{2}$, we obtain

$$
\begin{aligned}
& g^{\mu \nu} S_{B, \mathrm{TT}}^{\alpha \beta} \operatorname{Tr}\left[\not{ }_{d} G_{\nu \beta} \not h \hat{G}_{\mu \alpha}\right] \\
& =-\frac{32 Q^{2} q_{T}^{2}}{\sqrt{2} s^{5 / 2} x_{a}\left(\tau-x_{a} x_{2}\right)^{2}} \cos (2 \phi) .
\end{aligned}
$$

Substituting Eq. (96) into Eq. (82), we finally obtain the cross section as

$$
\begin{aligned}
\frac{d \sigma_{p d \rightarrow \mu^{+} \mu^{-} X}}{d \tau d q_{T}^{2} d \phi d y}\left(E_{x}-E_{y}\right)= & -\frac{\alpha^{2} \alpha_{s} C_{F} q_{T}^{2}}{6 \pi s^{3}} \cos (2 \phi) \int_{\min \left(x_{a}\right)}^{1} d x_{a} \frac{1}{\left(x_{a} x_{b}\right)^{2}\left(x_{a}-x_{1}\right)\left(\tau-x_{a} x_{2}\right)^{2}} \\
& \times \sum_{q} e_{q}^{2} x_{a}\left[q_{A}\left(x_{a}\right)+\bar{q}_{A}\left(x_{a}\right)\right] x_{b} \Delta_{T} g_{B}\left(x_{b}\right) .
\end{aligned}
$$

Here, the deuteron polarization is explicitly written as $E_{x}-E_{y}$, which indicates the polarization asymmetry of Eq. (53).

Actual polarization measurements are usually done by polarization asymmetries. For such estimations, the cross section $d \sigma_{p d \rightarrow \mu^{+} \mu^{-} X}\left(E_{x}+E_{y}\right)$ should be calculated in the same way. There are two process types, $q \bar{q} \rightarrow \gamma^{*} g$ and $q($ or $\bar{q}) g \rightarrow \gamma^{*} q($ or $\bar{q})$. For the $q \bar{q} \rightarrow \gamma^{*} g$ processes, the hadron tensor, which corresponds to Eq. (85), is given by

$$
\begin{aligned}
W_{\mu \nu}\left(q \bar{q}, E_{x}+E_{y}\right)= & \sum_{\lambda_{d}} \sum_{\text {color }} \sum_{q} e_{q}^{2} \int_{\min \left(x_{a}\right)}^{1} d x_{a} \frac{\pi}{x_{a}-x_{1}} \operatorname{Tr}\left[\Gamma_{q \bar{q} \rightarrow \gamma^{*} g, \nu} \Phi_{q / A}\left(x_{a}\right) \hat{\Gamma}_{q \bar{q} \rightarrow \gamma^{*} g, \mu} \Phi_{\bar{q} / B}\left(x_{b}\right)\right. \\
& \left.+\Gamma_{\bar{q} q \rightarrow \gamma^{*} g, \nu} \Phi_{\bar{q} / A}\left(x_{a}\right) \hat{\Gamma}_{\bar{q} q \rightarrow \gamma^{*} g, \mu} \Phi_{q / B}\left(x_{b}\right)\right] .
\end{aligned}
$$

For the $q($ or $\bar{q}) g \rightarrow \gamma^{*} q($ or $\bar{q})$ processes, the hadron tensor, which corresponds to Eq. (86), is given by

$$
\begin{aligned}
W_{\mu \nu}\left(q g, E_{x}+E_{y}\right)= & \sum_{\lambda_{d}} \sum_{\text {color }} \sum_{q} e_{q}^{2} \int_{\min \left(x_{a}\right)}^{1} d x_{a} \frac{\pi}{x_{a}-x_{1}} \operatorname{Tr}\left[\frac{1}{p_{g}^{-}} \Gamma_{q g \rightarrow \gamma^{*} q, \nu \beta}\left\{\Phi_{q / A}\left(x_{a}\right)+\Phi_{\bar{q} / A}\left(x_{a}\right)\right\} \hat{\Gamma}_{q g \rightarrow \gamma^{*} q, \mu \alpha} \Phi_{g / B}^{\alpha \beta}\left(x_{b}\right)\right. \\
& \left.+\frac{1}{p_{g}^{+}} \Gamma_{g q \rightarrow \gamma^{*} q, \nu \beta}\left\{\Phi_{q / B}\left(x_{b}\right)+\Phi_{\bar{q} / B}\left(x_{b}\right)\right\} \hat{\Gamma}_{g q \rightarrow \gamma^{*} q, \mu \alpha} \Phi_{g / A}^{\alpha \beta}\left(x_{a}\right)\right] .
\end{aligned}
$$

Here, the linear polarizations $E_{x}$ and $E_{y}$ are considered for the deuteron. For the polarization $E_{x}\left(E_{y}\right)$, the spin vector and tensor of Eq. (31) are given by $\vec{S}=0$ and $T_{i j}=$ $\delta_{i j} / 3-\delta_{i 1} \delta_{j 1}\left(T_{i j}=\delta_{i j} / 3-\delta_{i 2} \delta_{j 2}\right)$, which indicate $S_{B, T}^{x}=$ $S_{B, T}^{y}=S_{B, L}=S_{B, \mathrm{TT}}^{x y}=S_{B, L T}^{x}=S_{B, L T}^{y}=0, \quad S_{B, L L}=1 / 2$, and $S_{B, \mathrm{TT}}^{x x}=-1\left(S_{B, \mathrm{TT}}^{x x}=1\right.$ for $\left.E_{y}\right)$ in Eq. (30) for the deuteron $(B)$. Then, the correlation functions of Eqs. (66) and (67) for the deuteron become

$$
\begin{aligned}
& \Phi_{q / B}\left(x_{b}\right)_{E_{x}}+\Phi_{q / B}\left(x_{b}\right)_{E_{y}} \\
& \quad=\not h f_{1, q / B}\left(x_{b}\right)+\not h S_{B, L L} f_{1 L L, q / B}\left(x_{b}\right), \\
& \Phi_{g / B}^{\alpha \beta}\left(x_{b}\right)_{E_{x}}+\Phi_{g / B}^{\alpha \beta}\left(x_{b}\right)_{E_{y}} \\
& \quad=-g_{T}^{\alpha \beta} f_{1, g / B}\left(x_{b}\right)-g_{T}^{\alpha \beta} S_{B, L L} f_{1 L L, g / B}\left(x_{b}\right) .
\end{aligned}
$$

Therefore, the collinear quark and gluon correlation functions are expressed by the unpolarized PDFs $f_{1, q / B}\left(x_{b}\right)$ and $f_{1, g / B}\left(x_{b}\right)$ and the tensor-polarized PDFs $f_{1 L L, q / B}\left(x_{b}\right)$ and $f_{1 L L, g / B}\left(x_{b}\right)$. Although there is some information of the tensor-polarized quark distributions $f_{1 L L, q / B}\left(x_{b}\right)$ from the HERMES measurement [31,34], there are no established distributions at this stage. A finite tensor-polarized gluon distribution should appear through the $Q^{2}$ evolution [33]; however, there is no reliable distribution at this stage. In any case, the tensor-polarized PDFs are of the order of a few percent or less in comparison with the unpolarized PDFs $[31,33,34]$, they are neglected in numerical analysis of this work by taking $\Phi_{q / B}\left(x_{b}\right)_{E_{x}}+\Phi_{q / B}\left(x_{b}\right)_{E_{y}}=\not h f_{1, q / B}\left(x_{b}\right)$ and $\Phi_{g / B}^{\alpha \beta}\left(x_{b}\right)_{E_{x}}+\Phi_{g / B}^{\alpha \beta}\left(x_{b}\right)_{E_{y}}=-g_{T}^{\alpha \beta} f_{1, g / B}\left(x_{b}\right)$.

Repeating similar calculations for the cross section, we obtain 


$$
\begin{aligned}
\frac{d \sigma_{p d \rightarrow \mu^{+} \mu^{-} X}}{d \tau d q_{T}^{2} d \phi d y}\left(E_{x}+E_{y}\right)= & \frac{\alpha^{2} \alpha_{s} C_{F}}{2 \pi Q^{2}} \int_{\min \left(x_{a}\right)}^{1} d x_{a} \frac{1}{x_{a}-x_{1}} \sum_{q} e_{q}^{2}\left[\frac{4}{9}\left\{q_{A}\left(x_{a}\right) \bar{q}_{B}\left(x_{b}\right)+\bar{q}_{A}\left(x_{a}\right) q_{B}\left(x_{b}\right)\right\} \frac{2 Q^{2} \hat{s}+\hat{t}^{2}+\hat{u}^{2}}{\hat{s} \hat{t} \hat{u}}\right. \\
& \left.-\frac{1}{6 \hat{s}}\left(\left\{q_{A}\left(x_{a}\right)+\bar{q}_{A}\left(x_{a}\right)\right\} g_{B}\left(x_{b}\right) \frac{2 Q^{2} \hat{u}+\hat{s}^{2}+\hat{t}^{2}}{\hat{s} \hat{t}}+g_{A}\left(x_{a}\right)\left\{q_{B}\left(x_{b}\right)+\bar{q}_{B}\left(x_{b}\right)\right\} \frac{2 Q^{2} \hat{t}+\hat{s}^{2}+\hat{u}^{2}}{\hat{s} \hat{u}}\right)\right] .
\end{aligned}
$$

In fact, this expression agrees with the one in Ref. [55]. There is an overall factor of 2 in Eq. (101) because $d \sigma\left(E_{x}\right)+$ $d \sigma\left(E_{x}\right)$ is calculated instead of the spin average in Ref. [55]. The cross section is expressed by the variables $x_{a}, x_{b}, x_{1}, x_{2}$ as

$$
\begin{aligned}
\frac{d \sigma_{p d \rightarrow \mu^{+} \mu^{-} X}}{d \tau d q_{T}^{2} d \phi d y}\left(E_{x}+E_{y}\right)= & \frac{\alpha^{2} \alpha_{s} C_{F}}{2 \pi \tau s^{2}} \int_{\min \left(x_{a}\right)}^{1} d x_{a} \frac{1}{\left(x_{a}-x_{1}\right) x_{a}^{2} x_{b}^{2}} \\
& \times \sum_{q} e_{q}^{2}\left[\frac{4}{9}\left\{q_{A}\left(x_{a}\right) \bar{q}_{B}\left(x_{b}\right)+\bar{q}_{A}\left(x_{a}\right) q_{B}\left(x_{b}\right)\right\}\right. \\
& \times \frac{2 \tau\left\{\tau-\left(-2 x_{a} x_{b}+x_{1} x_{b}+x_{2} x_{a}\right)\right\}+x_{b}^{2}\left(x_{a}-x_{1}\right)^{2}+x_{a}^{2}\left(x_{b}-x_{2}\right)^{2}}{\left(x_{a}-x_{1}\right)\left(x_{b}-x_{2}\right)} \\
& +\frac{1}{6}\left\{q_{A}\left(x_{a}\right)+\bar{q}_{A}\left(x_{a}\right)\right\} g_{B}\left(x_{b}\right) \frac{2 \tau\left(\tau-x_{1} x_{b}\right)+x_{b}^{2}\left\{\left(x_{a}-x_{1}\right)^{2}+x_{a}^{2}\right\}}{x_{b}\left(x_{a}-x_{1}\right)} \\
& \left.+\frac{1}{6} g_{A}\left(x_{a}\right)\left\{q_{B}\left(x_{b}\right)+\bar{q}_{B}\left(x_{b}\right)\right\} \frac{2 \tau\left(\tau-x_{2} x_{a}\right)+x_{a}^{2}\left\{\left(x_{b}-x_{2}\right)^{2}+x_{b}^{2}\right\}}{x_{a}\left(x_{b}-x_{2}\right)}\right] .
\end{aligned}
$$

Then, the polarization asymmetry

$$
A_{E_{x y}}=\frac{d \sigma_{p d \rightarrow \mu^{+} \mu^{-} X}\left(E_{x}-E_{y}\right) /\left(d \tau d q_{T}^{2} d \phi d y\right)}{d \sigma_{p d \rightarrow \mu^{+} \mu^{-} X}\left(E_{x}+E_{y}\right) /\left(d \tau d q_{T}^{2} d \phi d y\right)}
$$

is estimated numerically in Sec. IV.

\section{NUMERICAL RESULTS}

By the formula in Eq. (97), the cross section is evaluated numerically. We need two types of parton distributions for this calculation. One is the unpolarized PDFs $q_{A}$ and $\bar{q}_{A}$ in the proton, and the other is the gluon transversity $\Delta_{T} g_{B}$ in the deuteron. Since the unpolarized PDFs are accurately determined except for extreme kinematical regions, if one of any recent parametrizations is taken, it provides a reasonable estimate on the proton part. As one of such a parametrization, the LO set of the CTEQ14 [56] is used for our numerical calculations in the leading-order and leadingtwist level. There is a charge squared factor in Eq. (97), so that the proton part simply becomes the structure function $F_{2}$ in the LO.

Next, an appropriate gluon transversity distribution should be taken. However, due to the lack of experimental information, there is no realistic gluon transversity distribution for evaluating the cross section. Since it is the purpose of this work to propose a possible experiment to find it at hadron accelerator facilities, we need to have a rough estimate on the magnitude of the cross section for future experimental proposals. We note that the quark transversity distributions in the proton are expected to be equal to the longitudinally polarized quark distributions at small $Q^{2}$, where the longitudinal polarization and the transverse one do not matter. From this consideration, we may boldly assume at first that the gluon transversity distribution is the same as the longitudinally polarized gluon distribution for calculating the cross section, just as a rough order of magnitude estimate on the cross section. However, we should note that the longitudinally polarized gluon distribution and the gluon transversity distribution have different physics origins, so that the actual cross section would be different. Of course, such an assumed distribution should be actually measured by future experiments. Later, we show how the cross section depends on this assumption.

The longitudinally polarized gluon distribution is not well determined at this stage, and it is one of the major purposes of building the future electron-ion collider. We take one of recent parametrizations, obtained by global analysis of world data in polarized proton reactions, on the longitudinally polarized gluon distribution. The employed set is the neural network (NN) PDFpoll.1 version [57]. The NNPDFpol gluon distributions are shown in Fig. 11 at $Q^{2}=20,30$, and $50 \mathrm{GeV}^{2}$. Since the dimuon cross sections are measured in the Fermilab Drell-Yan experiment in the dimuon-mass region, $4^{2}<M_{\mu \mu}^{2}=Q^{2}<9^{2} \mathrm{GeV}^{2}$, we consider the scale dependence in this range. The NNPDF determination reflects the Relativistic Heavy Ion Collider 


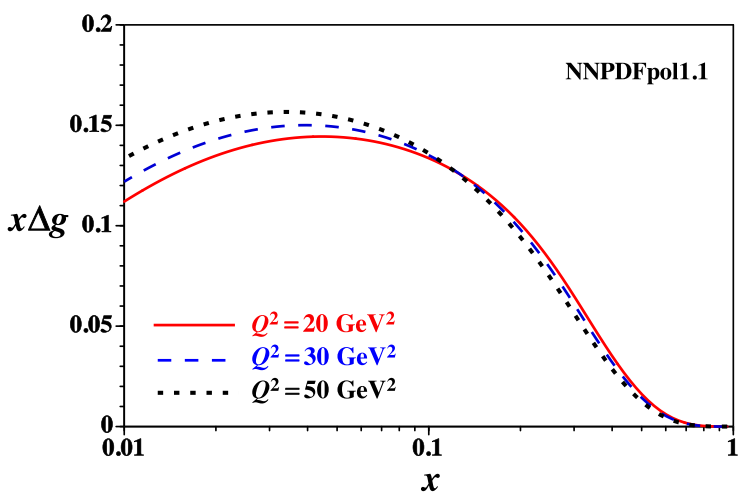

FIG. 11. Used longitudinally polarized gluon distributions of the NNPDFpol1.1 parametrization are shown at $Q^{2}=20,30$, and $50 \mathrm{GeV}^{2}$. These gluon distributions are used for a rough estimate on the Drell-Yan cross sections.

(RHIC) pion- and jet-production measurements, which are sensitive to the polarized gluon distribution, and it is one of reliable models at this stage.

There are two factors which need to be carefully considered in using the nucleonic PDFs for the deuteron ones. The first point is to assume that the deuteron PDFs are simply given by the addition of proton and neutron contributions as the first approximation. In our case, the gluon transversity of the deuteron is tentatively assumed as the addition of the longitudinally polarized gluon distributions for the proton and neutron, $\Delta_{T} g_{d}=\Delta g_{p}+\Delta g_{n}$.

The second point is that one needs to be careful about the scaling variable or the momentum fraction, as discussed after Eq. (85). In the Fermilab Drell-Yan experiment, the momentum fraction for a parton $q$ in the deuteron is defined by $p_{q}=x_{2}\left(p_{d} / 2\right)$, namely by using the deuteron momentum per nucleon, in Fermilab Drell-Yan experiments [44], so that its kinematical range is, in principle, $0 \leq x_{2} \leq 2$. However, the range $1 \leq x_{2} \leq 2$ is usually neglected and it is not even shown because the PDFs are tiny and it cannot be reached by experiments $[26,44]$. This definition of $x_{2}$ corresponds to the Bjorken scaling variable $x=$ $Q^{2} /\left(2 M_{N} \nu\right)(0 \leq x \leq 2)$ used in lepton DIS experiments with the deuteron target.

The momentum fraction $x_{b}$ has been used in this paper for partons in the deuteron, and its range is given by $0 \leq$ $x_{b} \leq 1$ by definition. If deuteron structure functions are assumed to be a simple addition of proton and neutron ones, finite PDFs exist only in the kinematical region of $0 \leq x_{b} \leq 1 / 2$ and they vanish in the region $1 / 2 \leq x_{b} \leq 1$. On the other hand, the nucleonic PDFs are provided in the Bjorken- $x$ region of $0 \leq x_{B j} \leq 1$, and the deuteron PDFs exist in the range $0 \leq x_{B j} \leq 2$. Since the cross section is formulated by using the variable $x_{b}$, we need to express $\Delta_{T} g_{d}\left(x_{b}\right)$ in Eq. (97) in terms of $\Delta g_{p, n}\left(x_{B j}\right)$ for the proton and neutron. To preserve the parton densities in changing the scaling variable, we need to take $\Delta_{T} g_{d}\left(x_{b}\right)=$ $2\left[\Delta g_{p}\left(x_{B j}\right)+\Delta g_{n}\left(x_{B j}\right)\right]$, where $0 \leq x_{B j} \leq 1$ for the nucleon, in evaluation of the cross section. For calculating this relation in the cross section, we first define the momentum fraction $x_{2}$, in the range $0 \leq x_{2} \leq 2$, by $x_{2} \equiv 2 x_{b}$ from $x_{b}$ which is given by $x_{a}$ in Eq. (79). Then, the only range $0 \leq x_{2} \leq 1$ is used as the scaling variable $x_{B j}$ for the nucleons. Alternatively, one can formulate the Drell-Yan cross section from the beginning by the independent addition of proton-proton and proton-neutron cross sections [58] to check this kinematical factor of 2. We actually confirmed such a factor of 2 , for example, by considering the simple subprocess $q \bar{q} \rightarrow \gamma^{*} \rightarrow \mu^{+} \mu^{-}$for the unpolarized Drell-Yan process. In fact, such a formalism has been used in the proposals of the Fermilab Drell-Yan experiments with the definition of the c.m. energy of Eq. (42).

Finally, we show the cross sections obtained by using the CTEQ14 for the proton PDFs and the gluon transversity distribution, which is assumed to be the longitudinal one in Fig. 11, as the function of the dimuon-mass squared $\left(M_{\mu \mu}^{2}=Q^{2}\right)$ in Fig. 12. The dimuon azimuthal angle and the dimuon rapidity are fixed at $\phi=0$ and $y=0.5$. The dotted, solid, and dashed curves indicate the cross sections at $q_{T}=0.2,0.5$, and $1.0 \mathrm{GeV}$, respectively. The hard scale $Q^{2}$ for calculating the PDFs is taken as the dimuon-mass squared $Q^{2}=M_{\mu \mu}^{2}$. The magnitude of the cross section is typically $0.001 \sim 0.1 \mathrm{nb} / \mathrm{GeV}^{2}$ in this kinematical range. The dependence of the cross section on the dimuon transverse-momentum $q_{T}$ is shown in Fig. 13. The solid, dashed, and dotted curves indicate the cross sections at $M_{\mu \mu}^{2}=20,30$, and $50 \mathrm{GeV}^{2}$, respectively. In Fig. 14, the dependence is shown on the dimuon rapidity at $q_{T}=0.2$,

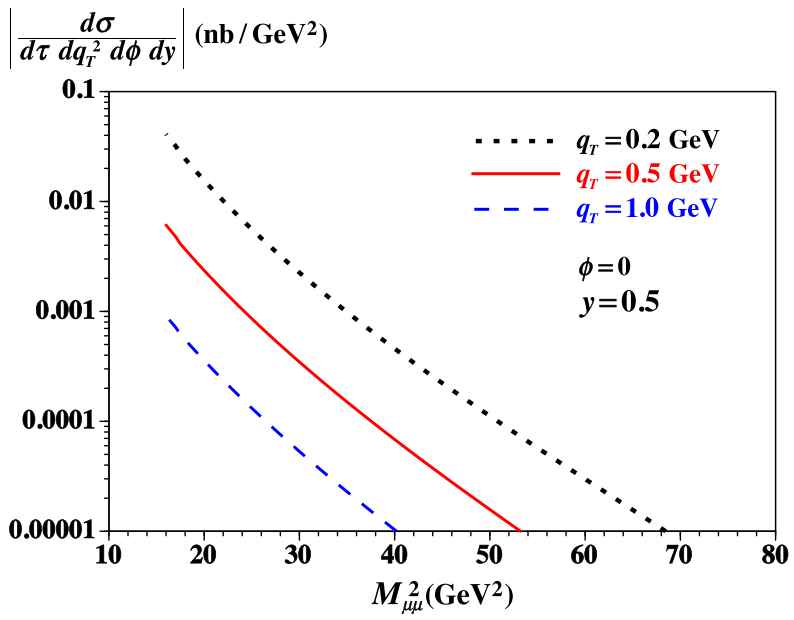

FIG. 12. The proton-deuteron Drell-Yan cross sections are shown as the function of the dimuon-mass squared $M_{\mu \mu}^{2}$ at $q_{T}=0.2,0.5$, and $1 \mathrm{GeV}$ for the dimuon azimuthal angle $\phi=0$ and the dimuon rapidity $y=0.5$. The CTEQ14 PDFs are used for the unpolarized PDFs of the proton, and the longitudinally polarized gluon distribution is taken from the NNPDF1.1, and the gluon transversity is assumed to be the same as the longitudinal one for numerical estimates. 


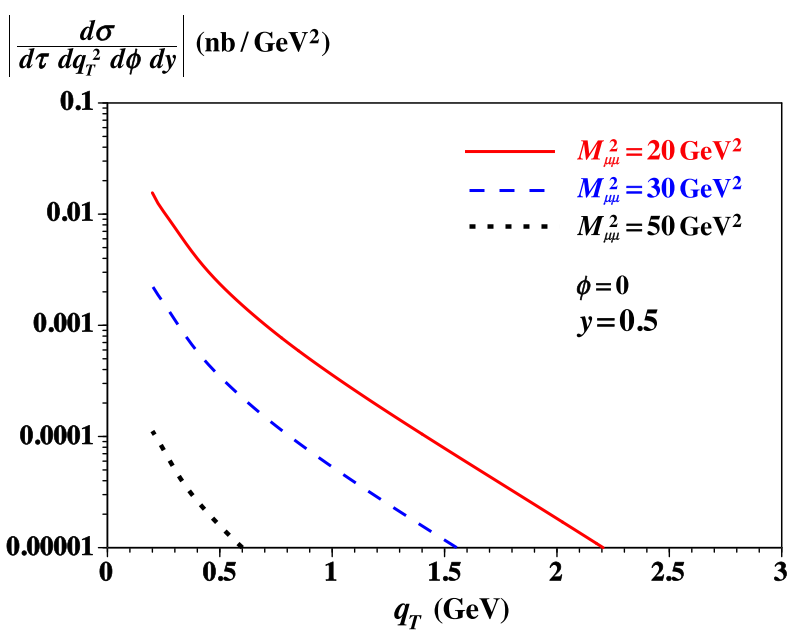

FIG. 13. The proton-deuteron Drell-Yan cross sections are shown as the function of the dimuon transverse momentum $q_{T}$ at the dimuon-mass squared $M_{\mu \mu}^{2}=20,30$, and $50 \mathrm{GeV}^{2}$ for the azimuthal angle $\phi=0$ and the rapidity $y=0.5$.

0.5 , and $1.0 \mathrm{GeV}$ by fixing the angle $\phi=0$ and the dimuon-mass squared $M_{\mu \mu}^{2}=20 \mathrm{GeV}^{2}$.

In Figs. 12 and 13, the cross section drops fast as $p_{T}$ and $M_{\mu \mu}^{2}\left(=Q^{2}\right)$ increase. The kinematical factor in the cross section integrand of Eq. (97) and the integral minimum for $x_{a}$ are given by

$$
\begin{aligned}
& \frac{q_{T}^{2}}{x_{a} x_{b}\left(x_{a}-x_{1}\right)\left(\tau-x_{a} x_{2}\right)^{2}}=\frac{q_{T}^{2}}{x_{a}\left(\frac{Q^{2}}{s}-x_{a} \sqrt{\frac{Q^{2}+q_{T}^{2}}{s}} e^{-y}\right)^{3}}, \\
& \min \left(x_{a}\right)=\frac{x_{1}-\tau}{1-x_{2}}=\frac{\sqrt{\frac{Q^{2}+q_{T}^{2}}{s}} e^{y}-\frac{Q^{2}}{s}}{1-\sqrt{\frac{Q^{2}+q_{T}^{2}}{s}} e^{-y}} .
\end{aligned}
$$

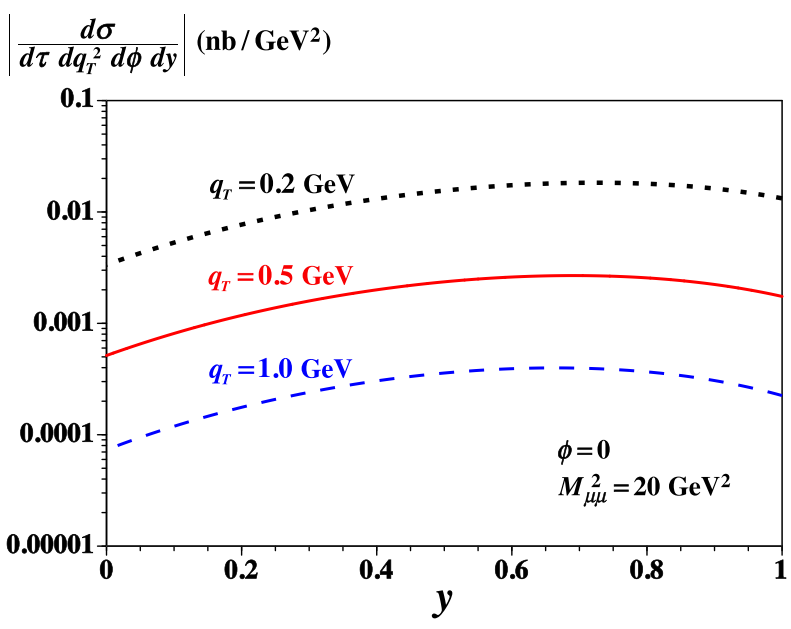

FIG. 14. The proton-deuteron Drell-Yan cross sections are shown as the function of the dimuon rapidity $y$ at the dimuon transverse momentum $q_{T}=0.2,0.5$, and $1 \mathrm{GeV}$ for the azimuthal angle $\phi=0$ and the dimuon-mass squared $M_{\mu \mu}^{2}=20 \mathrm{GeV}^{2}$.
The first equation indicates that the cross section decreases with $Q^{2}$ and $q_{T}$. In addition, the minimum of $x_{a}$ increases with $Q^{2}$ and $q_{T}$ at positive rapidity $y$, which restricts the integral region. Since there is a factor $1 /\left(x_{a}\right)^{2}$ in the integrand, the increase of $Q^{2}$ and $q_{T}$ significantly reduces the cross section. Third, the scale $Q^{2}$ is given by $M_{\mu \mu}^{2}$ and the PDFs, $q_{A}\left(x_{a}\right), \bar{q}_{A}\left(x_{a}\right)$, and $\Delta_{T} g_{B}\left(x_{b}\right)$, change with $Q^{2}$. Furthermore, the running coupling constant $\alpha_{s}$ also becomes slightly smaller by the increase of $Q^{2}$.

In Figs. 12-14, the gluon transversity in the deuteron is assumed to be $\Delta_{T} g=\Delta g_{p}+\Delta g_{n}$ for estimating the cross sections. However, it is just an assumption. As mentioned before, the order of magnitude of the quark transversity distributions are expected to be similar to the longitudinally polarized ones, whereas the gluon transversity in the deuteron would be very different from the longitudinally polarized gluon distribution in the nucleon. In Fig. 15, we show the cross sections also by taking $\Delta_{T} g=\left(\Delta g_{p}+\right.$ $\left.\Delta g_{n}\right) / 2$ or $\left(\Delta g_{p}+\Delta g_{n}\right) / 4$. One should note that the actual cross sections could be very different from the estimates by using the assumed gluon transversity $\Delta_{T} g=\Delta g_{p}+\Delta g_{n}$.

So far, we have shown the absolute cross sections. However, actual measurements will be done in polarization asymmetries, and their numerical results are shown in Fig. 16 for the polarization asymmetry defined in Eq. (103) as the function of the dimuon-mass squared $M_{\mu \mu}^{2}$. Since the gluon transversity distributions are assumed to be the same as the longitudinally polarized ones in this figure, the numerical values are likely to be most optimistic ones. The

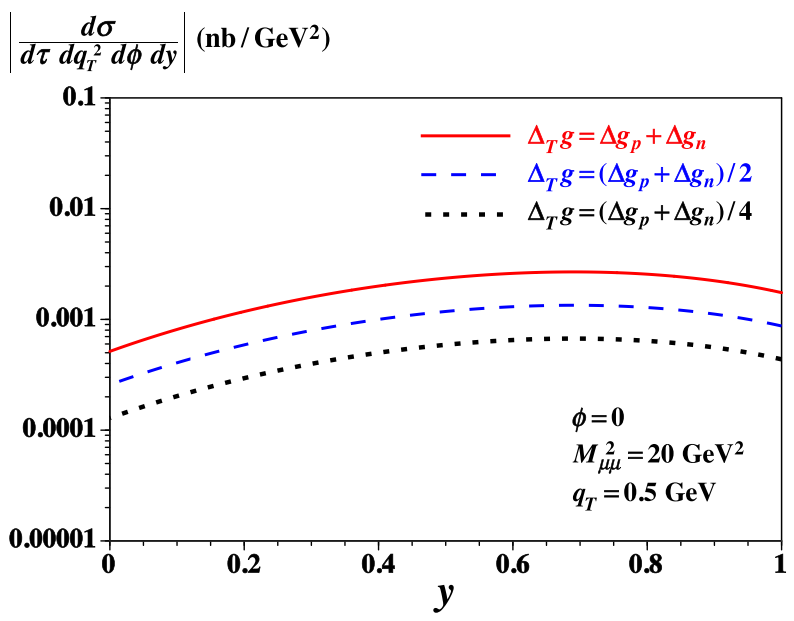

FIG. 15. Dependence is shown on the choice of the gluon transversity for the proton-deuteron Drell-Yan cross section as the function of the rapidity $y$ at the dimuon transverse momentum $q_{T}=0.5 \mathrm{GeV}$ for the azimuthal angle $\phi=0$ and the dimuonmass squared $M_{\mu \mu}^{2}=20 \mathrm{GeV}^{2}$. The NNPDF1.1 gluon distribution is used. Three gluon transversity distributions are assumed as $\Delta_{T} g=\Delta g_{p}+\Delta g_{n},\left(\Delta g_{p}+\Delta g_{n}\right) / 2$, and $\left(\Delta g_{p}+\Delta g_{n}\right) / 4$, where $p$ and $n$ indicate proton and neutron, respectively, for calculating the cross section. 


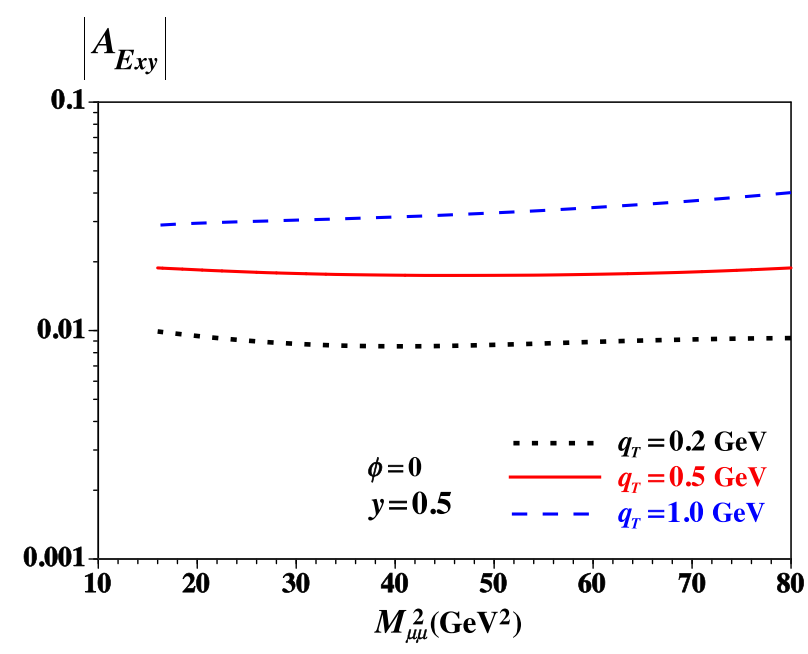

FIG. 16. The spin asymmetries $\left|A_{E_{x y}}\right|$ for the proton-deuteron Drell-Yan cross sections are shown as the function of the dimuonmass squared $M_{\mu \mu}^{2}$ at $q_{T}=0.2,0.5$, and $1 \mathrm{GeV}$ for the dimuon azimuthal angle $\phi=0$ and the dimuon rapidity $y=0.5$. The CTEQ14 PDFs are used for the unpolarized PDFs of the proton and the deuteron. The longitudinally polarized gluon distribution is taken from the NNPDF1.1, and the gluon transversity is assumed to be the same as the longitudinal one for numerical estimates.

asymmetries are likely to be smaller than these vales. However, the asymmetries could be within the reach of future experimental measurements.

The measurement of the gluon transversity is considered at JLab [25]. In the studies of nucleon structure functions, the measurements of hadron-accelerator facilities have been often complementary and much better in some aspects of the PDFs. For example, the pion- and jet-production measurement in polarized proton-proton collisions at RHIC provided a constraint on the longitudinally polarized gluon distribution, namely the gluon-spin contribution to the nucleon spin. Furthermore, the typical $Q^{2}$ range $\left(20<Q^{2}<50 \mathrm{GeV}^{2}\right)$ of the Drell-Yan experiment is much higher than the JLab measurements at a few to several $\mathrm{GeV}^{2}$. In the similar way, it is a good idea to propose independent experiments to measure the gluon transversity at hadron facilities. With this motivation, we proposed the Drell-Yan measurement in the protondeuteron reaction with the polarized deuteron. It needs the linearly polarized deuteron with the unpolarized proton beam, and the azimuthal-angle information [59] is necessary for the dimuon in the final state. The cross section is typically $0.001-0.1 \mathrm{nb} / \mathrm{GeV}^{2}$, so that it may not be an easy experiment. However, there are available hadron facilities at Fermilab, J-PARC (Japan Proton Accelerator Research Complex), GSI-FAIR (Gesellschaft für
Schwerionenforschung -Facility for Antiproton and Ion Research), and NICA (Nuclotron-based Ion Collider fAcility). In addition, if the fixed-deuteron target becomes possible at RHIC, Large Hadron Collider, or EIC, there could be a possibility. We hope that our theoretical proposal is realized in future experiments at some facility.

\section{SUMMARY}

Instead of the longitudinally polarized parton distribution functions, the nucleon spin structure can be investigated by the transversely polarized ones. The leadingtwist parton-distribution functions in the transversely polarized nucleon are quark transversity distributions, which have chiral-odd nature, and there is some experimental information. On the other hand, the gluon transversity does not exist for the spin- $1 / 2$ nucleon because the two unit of spin flip $(\Delta s=2)$ is necessary. It exists for the spin-1 deuteron. There is an experimental project to measure the gluon transversity by electron scattering; however, it is valuable if it can be investigated at hadron-accelerator facilities, as an independent experiment and to probe the different kinematical region. There was no theoretical proposal to find it in hadron reactions before this work.

In this paper, the possibility was proposed to find the gluon transversity at hadron accelerator facilities, especially, in the proton-deuteron reactions. We found that it is possible in the proton-deuteron Drell-Yan process with the linearly polarized deuteron. For the final dimuon, the experimental measurement on the azimuthal-angle distribution is necessary. We showed expected dependencies of the cross section on the dimuon-mass squared $M_{\mu \mu}^{2}$, the dimuon transverse-momentum $p_{T}$, and the dimuon rapidity $y$, and the assumption on the magnitudes of the gluon transversity $\Delta_{T} g$. The order of cross section was typically estimated as $0.001-0.1 \mathrm{nb} / \mathrm{GeV}^{2}$. Then, the spin asymmetries were shown and they could be within the reach of experimental measurements. Hopefully, it will be realized in future experiments at hadron facilities in addition to the electron scattering experiment at JLab and possibly at EIC.

\section{ACKNOWLEDGMENTS}

The authors thank W.-C. Chang, W. Detmold, D. Keller, P. Mulders, K. Nakano, J. Qiu, K. Tanaka, W. Vogelsang, and S. Yoshida for suggestions. This work was partially supported by Japan Society for the Promotion of Science Grants-in-Aid for Scientific Research (KAKENHI) Grant No. 19K03830. Q.-T.S is supported by the MEXT Scholarship for foreign students through the Graduate University for Advanced Studies. 
[1] M. Gell-Mann, Phys. Lett. 8, 214 (1964); G. Zweig, CERN Reports No. 8182/TH.401 and No.8419/TH.412, 1964.

[2] J. Ashman et al. (European Muon Collaboration), Phys. Lett. B 206, 364 (1988).

[3] For review, see S. E. Kuhn, J.-P. Chen, and E. Leader, Prog. Part. Nucl. Phys. 63, 1 (2009); A. Deur, S. J. Brodsky, and G. F. de Teramond, Rep. Prog. Phys. 82, 076201 (2019), and references therein.

[4] E. Leader and C. Lorce, Phys. Rep. 541, 163 (2014); M. Wakamatsu, Int. J. Mod. Phys. A 29, 1430012 (2014).

[5] M. Deka et al., Phys. Rev. D 91, 014505 (2015); C. Alexandrou, M. Constantinou, K. Hadjiyiannakou, K. Jansen, C. Kallidonis, G. Koutsou, A. V. Avilés-Casco, and C. Wiese, Phys. Rev. Lett. 119, 142002 (2017).

[6] X. Ji, Phys. Rev. Lett. 110, 262002 (2013); for recent progress, see, e.g., T. Ishikawa, Y.-Q. Ma, J.-W. Qiu, and S. Yoshida, Phys. Rev. D 96, 094019 (2017); H.-W. Lin et al., Prog. Part. Nucl. Phys. 100, 107 (2018); Yu.-S. Liu et al., Phys. Rev. D 101, 034020 (2020); for recent progress, see the file of J. Zhang, 11th Circum-Pan-Pacific Symposium on High Energy Spin Physics, Miyazaki, Japan (2019), https:// indico2.riken.jp/event/3039/.

[7] M. Diehl, Phys. Rep. 388, 41 (2003); S. Wallon, Doctoral school lecture notes on courses ED-107 and ED-517, Université Paris Sud, 2014 (unpublished), http://pperso.th .u-psud.fr/page_perso/Wallon/cours/exclusif.pdf.

[8] K. Goeke, M. V. Polyakov, and M. Vanderhaeghen, Prog. Part. Nucl. Phys. 47, 401 (2001); X. Ji, Annu. Rev. Nucl. Part. Sci. 54, 413 (2004); A. V. Belitsky and A. V. Radyushkin, Phys. Rep. 418, 1 (2005); S. Boffi and B. Pasquini, Riv. Nuovo Cimento 30, 387 (2007); M. Diehl and P. Kroll, Eur. Phys. J. C 73, 2397 (2013); D. Mueller, FewBody Syst. 55, 317 (2014); K. Kumericki, S. Liuti, and H. Moutarde, Eur. Phys. J. A 52, 157 (2016); H. Moutarde, P. Sznajder, and J. Wagner, Eur. Phys. J. C 78, 890 (2018).

[9] S. Kumano, Q.-T. Song, and O. V. Teryaev, Phys. Rev. D 97, 014020 (2018).

[10] U. D’Alesio and F. Murgia, Prog. Part. Nucl. Phys. 61, 394 (2008); V. Barone, F. Bradamante, and A. Martin, Prog. Part. Nucl. Phys. 65, 267 (2010); C. A. Aidala, S. D. Bass, D. Hasch, and G. K. Mallot, Rev. Mod. Phys. 85, 655 (2013); M. G. Perdekamp and F. Yuan, Annu. Rev. Nucl. Part. Sci. 65, 429 (2015).

[11] S. Kumano, Proc. Sci., DIS2018 (2018) 245.

[12] J. P. Ralston and D. E. Soper, Nucl. Phys. B152, 109 (1979).

[13] X. Artru and M. Mekhfi, Z. Phys. C 45, 669 (1990).

[14] R. L. Jaffe and X. D. Ji, Nucl. Phys. B375, 527 (1992).

[15] X. Artru, 10th Rhodanien Seminar: The Spin in Physics Turin, Italy (2002), https://arxiv.org/abs/hep-ph/0207309.

[16] V. Barone, A. Drago, and P. G. Ratcliffe, Phys. Rep. 359, 1 (2002).

[17] V. Barone and R. G. Ratcliffe, Transverse Spin Physics (World Scientific, Singapore, 2003).

[18] Z.-B. Kang, A. Prokudin, P. Sun, and F. Yuan, Phys. Rev. D 93, 014009 (2016); M. Radici and A. Bacchetta, Phys. Rev. Lett. 120, 192001 (2018).

[19] F. Baldracchini, N. S. Cragie, V. Roberto, and M. Socolovsky, Fortsch. Phys. 30, 505 (1981); X. Artru and M. Mekhfi, Z. Phys. C 45, 669 (1990); S. Kumano and M. Miyama, Phys. Rev. D 56, R2504 (1997); A. Hayashigaki, Y.
Kanazawa, and Y. Koike, Phys. Rev. D 56, 7350 (1997); W. Vogelsang, Phys. Rev. D 57, 1886 (1998); M. Hirai, S. Kumano, and M. Miyama, Comput. Phys. Commun. 111, 150 (1998).

[20] W. Vogelsang, Acta Phys. Pol. B 29, 1189 (1998); https:// www.actaphys.uj.edu.pl/R/29/5/1189/pdf.

[21] A. Czarnecki and B. Krause, Phys. Rev. Lett. 78, 4339 (1997); M. B. Hecht and B. H. J. McKellar, Phys. Rev. C 60, 065202 (1999); M. B. Hecht, C. D. Roberts, and S. M. Schmidt, Phys. Rev. C 64, 025204 (2001); T. Liu, Z. Zhao, and H. Gao, Phys. Rev. D 97, 074018 (2018); For review and recent update, see M. Pospelov and A. Ritz, Ann. Phys. (Amsterdam) 318, 119 (2005); T. E. Chupp, P. Fierlinger, M. J. Ramsey-Musolf, and J. T. Sigh, Rev. Mod. Phys. 91, 015001 (2019).

[22] B. Pire and L. Szymanowski, Phys. Rev. Lett. 115, 092001 (2015); W. Cosyn and B. Pire, Phys. Rev. D 98, 074020 (2018).

[23] Z. Ye, N. Sato, K. Allada, T. Liu, J.-P. Chen, H. Gao, Z.-B. Kang, A. Prokudin, P. Sun, and F. Yuan, Phys. Lett. B 767, 91 (2017).

[24] A. Accardi et al., Eur. Phys. J. A 52, 268 (2016).

[25] R. L. Jaffe and A. Manohar, Phys. Lett. B 223, 218 (1989); J.P. Ma, C. Wang, and G. P. Zhang, arXiv:1306.6693, (unpublished), http://www.jlab.org/exp_prog/proposals/16/ LOI12-16-006.pdf; M. Jones et al., A Letter of Intent to Jefferson Lab PAC 44, Jefferson Lab Report No. LOI12-16006, 2016.

[26] Fermilab E1039 experiment, Letter of Intent Report No. P1039, 2013, https://www.fnal.gov/directorate/program_planning/ June2013PACPublic/P-1039_LOI_polarized_DY.pdf; D. Keller and K. Nakano (private communication); for the ongoing Fermilab E-906/SeaQuest experiment, http:// www.phy.anl.gov/mep/drell-yan/.

[27] M. Nzar and P. Hoodbhoy, Phys. Rev. D 45, 2264 (1992).

[28] W. Detmold and P. E. Shanahan, Phys. Rev. D 94, 014507 (2016); 95, 079902 (2017).

[29] L. L. Frankfurt and M. I. Strikman, Nucl. Phys. A405, 557 (1983).

[30] P. Hoodbhoy, R. L. Jaffe, and A. Manohar, Nucl. Phys. B312, 571 (1989); R. L. Jaffe and A. Manohar, Nucl. Phys. B321, 343 (1989).

[31] J.-P. Chen et al., Proposal to Jefferson Lab PAC-38, Jefferson Lab Report No. PR12-11-110, 2011; F. E. Close and S. Kumano, Phys. Rev. D 42, 2377 (1990); T.-Y. Kimura and S. Kumano, Phys. Rev. D 78, 117505 (2008); S. Kumano, Phys. Rev. D 82, 017501 (2010); J. Phys. Conf. Ser. 543, 012001 (2014).

[32] S. Hino and S. Kumano, Phys. Rev. D 59, 094026 (1999); 60, 054018 (1999).

[33] S. Kumano and Q.-T. Song, Phys. Rev. D 94, 054022 (2016).

[34] A. Airapetian et al. (HERMES Collaboration), Phys. Rev. Lett. 95, 242001 (2005); W. Cosyn, Yu.-B. Dong, S. Kumano, and M. Sargsian, Phys. Rev. D 95, 074036 (2017).

[35] G. A. Miller, Phys. Rev. C 89, 045203 (2014).

[36] Section 2-1-3 of C. Itzykson and J.-B. Zuber, Quantum Field Theory (McGraw-Hill Inc., New York, 1980); Section 4 of S. Weinberg, The Quantum Theory of Fields (Cambridge University Press, Cambridge, United Kingdom, 1995), Vol. I. 
[37] R. L. Jaffe, in The Spin Structure of the Nucleon: International School of Nucleon Structure, edited by B. Frois et al. (World Scientific, Singapore, 1997).

[38] E. Leader, Spin in Particle Physics (Cambridge University Press, Cambridge, United Kingdom, 2001).

[39] A. Bacchetta and P. J. Mulders, Phys. Rev. D 62, 114004 (2000).

[40] A. Bacchetta, Ph.D. Thesis, Vrije Universiteit Amsterdam, 2002.

[41] D. Boer, S. Cotogno, T. van Daal, P. J. Mulders, A. Signori, and, S. Cotogno, J. High Energy Phys. 10 (2016) 013.

[42] T. van Daal, arXiv:1612.06585; Ph.D. thesis, University of Groningen, 2018. In our work, the correlation functions defined in this van Daal's paper are used.

[43] R. D. Field, Applications of Perturbative QCD (AddisonWesley, Reading, MA, 1989); R. K. Ellis, W. J. Stirling, and B. R. Webber, QCD and Collider Physics (Cambridge University Press, Cambridge, United Kingdom, 1996).

[44] W.-C. Chang (private communication).

[45] X. Ji, Phys. Lett. B 289, 137 (1992).

[46] Sect. 7.5.5 in J. Collins, Foundations of Perturbative QCD (Cambridge University Press, Cambridge, United Kingdom, 2011).

[47] P. J. Mulders and J. Rodrigues, Phys. Rev. D 63, 094021 (2001).
[48] E. Sather and C. Schmidt, Phys. Rev. D 42, 1424 (1990).

[49] S. Meissner, A. Metz, and K. Goeke, Phys. Rev. D 76, 034002 (2007).

[50] G. Sterman et al., Rev. Mod. Phys. 67, 157 (1995).

[51] M. Tanabashi et al. (Particle Data Group), Phys. Rev. D 98, 030001 (2018).

[52] J. Qiu and G. Sterman, Nucl. Phys. B353, 137 (1991); for recent works, see H. Xing and S. Yoshida, Adv. High Energy Phys. 2019, 4825790 (2019).

[53] V. Shtabovenko, R. Mertig, and F. Orellana, Comput. Phys. Commun. 207, 432 (2016).

[54] M. Jamin and M. E. Lautenbacher, Comput. Phys. Commun. 74, 265 (1993).

[55] See Eqs. (8.14), (8.25), and (8.20) of E. Reya, Phys. Rep. 69, 195 (1981).

[56] S. Dulat, T.-J. Hou, J. Gao, M. Guzzi, J. Huston, P. Nadolsky, J. Pumplin, C. Schmidt, D. Stump, and C.-P. Yuan, Phys. Rev. D 93, 033006 (2016).

[57] E. R. Nocera, R. D. Ball, S. Forte, G. Ridolfi, and J. Rojo, Nucl. Phys. B887, 276 (2014).

[58] M. Anselmino, A. Efremov, and E. Leader, Phys. Rep. 261, 1 (1995).

[59] L. Y. Zhu et al., Phys. Rev. Lett. 99, 082301 (2007); 102, 182001 (2009); W.-C. Chang, R. E. McClellan, J.-C. Peng, and O. Teryaev, Phys. Rev. D 99, 014032 (2019). 